\title{
Bridging Molecular Genetics and Biomarkers in Lewy Body and Related Disorders
}

\author{
Gilbert J. Ho, ${ }^{1,2}$ Willie Liang, ${ }^{1,2}$ Masaaki Waragai, ${ }^{3}$ Kazunari Sekiyama, ${ }^{3}$ \\ Eliezer Masliah, ${ }^{1}$ and Makoto Hashimoto ${ }^{3}$ \\ ${ }^{1}$ Department of Neurosciences, University of California, San Diego, La Jolla, CA 92093-0624, USA \\ ${ }^{2}$ The Center for Memory and Aging, Poway, CA 92064, USA \\ ${ }^{3}$ Laboratory for Chemistry and Metabolism, Tokyo Metropolitan Institute for Neuroscience, Tokyo 183-8526, Japan
}

Correspondence should be addressed to Gilbert J. Ho, giho@ucsd.edu

Received 30 December 2010; Accepted 20 April 2011

Academic Editor: G. B. Frisoni

Copyright (c) 2011 Gilbert J. Ho et al. This is an open access article distributed under the Creative Commons Attribution License, which permits unrestricted use, distribution, and reproduction in any medium, provided the original work is properly cited.

\begin{abstract}
Recent advances have been made in defining the genetic and molecular basis of dementia with Lewy bodies (DLBs) and related neurodegenerative disorders such as Parkinson's disease (PD) and Parkinson's disease dementia (PDD) which comprise the spectrum of "Lewy body disorders" (LBDs). The genetic alterations and underlying disease mechanisms in the LBD overlap substantially, suggesting common disease mechanisms. As with the other neurodegenerative dementias, early diagnosis in LBD or even identification prior to symptom onset is key to developing effective therapeutic strategies, but this is dependent upon the development of robust, specific, and sensitive biomarkers as diagnostic tools and therapeutic endpoints. Recently identified mutations in the synucleins and other relevant genes in PD and DLB as well as related biomolecular pathways suggest candidate markers from biological fluids and imaging modalities that reflect the underlying disease mechanisms. In this context, several promising biomarkers for the LBD have already been identified and examined, while other intriguing possible candidates have recently emerged. Challenges remain in defining their correlation with pathological processes and their ability to detect DLB and related disorders, and perhaps a combined array of biomarkers may be needed to distinguish various LBDs.
\end{abstract}

\section{Introduction}

Over the past decade, dementia with Lewy bodies (DLBs) has arguably become the second most common form of neurodegenerative dementia behind Alzheimer's disease (AD). In addition to progressive decline in cognition, DLB is characterized by fluctuations in cognition with variations in attention and alertness, recurrent formed visual hallucinations, visuospatial dysfunction, and spontaneous parkinsonism. Often, DLB patients also exhibit neuroleptic sensitivity, transient loss of consciousness, falls, and rapid eye movement (REM-) sleep behavior disorder [1]. The clinical separation of DLB from other similar disorders is often difficult resulting in poor diagnostic accuracy, but the relative temporal co-occurrence of parkinsonian features with the typical DLB cognitive and behavioral symptoms such as visuospatial disturbance strongly suggests the diagnosis. Clinical presentation of DLB is also influenced by the amount of $\mathrm{AD}$ tau pathology, further complicating the diagnosis [2]. Approximately $20-40 \%$ of Parkinson's disease (PD) patients also eventually develop a progressive dementing illness designated as Parkinson's disease dementia (PDD) characterized by a frontal-subcortical clinical presentation [3]. DLB/Diffuse Lewy body disease (DLBD), Lewy body variant of $\mathrm{AD}(\mathrm{LBV})$, and $\mathrm{PDD}$ comprise an emerging spectrum of clinical phenotypes from relatively pure motor $\mathrm{PD}$ to the more predominant cognitive and behavioral disturbance observed in PDD and DLB, yet the reason for the variability remains unknown. Despite the heterogeneity of their clinical phenotypes, a significant neuropathological overlap is observed among these diseases, hence the term "Lewy body disorders" (LBDs) to collectively describe conditions in which Lewy bodies (LBs) and Lewy neurites (LNs) predominate as the hallmark histological lesions. Variation in the distribution of Lewy body pathology is present among LBDs, with more neocortical and limbic system LB in both 
DLB and PDD compared to the brains of PD patients without cognitive symptoms and greater neuronal loss in substantia nigra in PDD than DLB [4]. Yet, DLB and overlap disorders such as LBV, more so than PDD, have $\beta$-amyloid pathology and basal forebrain cholinergic deficit similar to $\mathrm{AD}$ patients [5].

As with other forms of dementia, the pathobiological changes in LBD likely occur decades prior to the onset of clinical symptoms and correspond to widespread irreversible neurodegeneration $[6,7]$. It is increasingly clear from the $\mathrm{AD}$ therapeutic experience that by the time widespread neuronal injury ensues, symptomatic cholinergic treatments are minimally effective at best, and disease-modifying therapeutic approaches in trials have thus far proven ineffective at altering disease course or in rescuing diseased brain $[8$, 9]. To demonstrate efficacy, any potential disease-modifying therapy in neurodegenerative dementia must be initiated prior to the expression of the clinical phenotype during the initial molecular pathogenetic events before irreversible neuronal damage has occurred. At present, accurately predicting those individuals at risk for developing neurodegenerative dementia is challenging, and this places greater urgency on developing earlier methods of disease detection. Critically important is not only distinguishing the LBD from $\mathrm{AD}$ and other forms of dementia, but also separating DLBs and other LBDs. Although there are many promising candidates for LBD, no biomarkers have yet been validated for clinical diagnostic use, and thus many opportunities exist to develop such tests. Here, we highlight from the perspective of how major genetic discoveries in the LBD and their corresponding biomolecular processes might translate into useful disease markers in biological fluids. Because of many common pathogenetic features among the LBDs, the emerging genetic influences found in PD have readily been translated to DLBs and other LBDs, providing clues to rational approaches for selecting future DLB and LBD biomarker targets for exploration. This paper will highlight several PD and DLB genes and their protein products as candidates for biological disease markers (Table 1).

\section{Amyloid and Tau in Lewy Body Disorders}

2.1. Amyloid Genetics and Biomarkers in LBD. A $\beta$, a key component of neuritic plaques in $\mathrm{AD}$ brain, is overproduced leading to various degrees of amyloid aggregation and synaptic and neuronal toxicity [10]. As indicated previously, amyloid pathology in the form of neuritic and diffuse plaques can be also found in varying degrees in the brain tissue of patients with DLB, which may interact with LB or synuclein pathology or influence the clinical features of LBDs $[4,11]$. The genetic mechanisms of $\mathrm{A} \beta$ overproduction in $\mathrm{AD}$ are well established; the $\beta \mathrm{APP}$ gene (chromosome 21 ), the first identified AD susceptibility gene, encodes a transmembranous protein ranging from 695 to 770 residues, which undergoes a process of regulated intramembranous proteolysis ultimately releasing $\mathrm{A} \beta$ peptides, primarily $\mathrm{A} \beta 42$ and $\mathrm{A} \beta 40$, as well as other fragments. $\mathrm{A} \beta$ is generated by the concerted action of $\beta$-secretase and $\gamma$-secretase complex, while the $\alpha$-secretase pathway precludes $\mathrm{A} \beta$ formation by cleaving $\beta$ APP at a site within the $A \beta$ sequence. Genetic analysis of early-onset familial AD cases revealed numerous mutations in the $\beta$ APP gene as well as presenilin 1 (PS1; chromosome 14) and presenilin 2 (PS2; chromosome 1) genes, all of which accelerate the processing of $\beta$ APP, leading to increased $\mathrm{A} \beta$ generation [12]. Specifically, the $\beta \mathrm{APP}$ KM670/671NL (Swedish) mutation affects the $\beta$-secretase site, A692G (Flemish) mutation alters the $\alpha$-secretase site, and both V717F (Indiana) and V717I (London) mutations affect the $\gamma$-secretase processing, leading to elevated $\mathrm{A} \beta$ levels. Also important in the Notch developmental signaling pathway which is analogous to $\beta$ APP processing, the presenilins are thought to be a component of the $\gamma$-secretase enzyme complex, which suggests that missense mutations in the presenilins mechanistically lead to accelerated $\beta$ APP processing to $\mathrm{A} \beta[13]$.

Therefore, the abnormal proteolytic cleavage of $\beta$ APP leads to elevated brain $\mathrm{A} \beta$ deposition, and as a result, diminished peripheral levels of $\mathrm{A} \beta$. Reflecting a shift from soluble $\mathrm{A} \beta$ to insoluble brain deposits, significant decreases in CSF $A \beta 42$ levels have been demonstrated in $\mathrm{AD}$ and more recently in DLB cases [14]. Parnetti et al. found that DLB, compared with PD, PDD, and AD patients, showed the lowest CSF levels of $A \beta 42$ and, when combined with CSF tau, differentiated DLB from PD and PDD, but not from AD [15]. Also, Spies and colleagues showed a greater decrease in $\mathrm{A} \beta 40$ in clinical DLB and vascular dementia patients compared with control levels and even with $\mathrm{AD}$. Differentiation of non-AD dementias such as vascular dementia and DLB was improved by comparing the ratio of $A \beta 42$ and $A \beta 40$ [16]. More recently, the detection of amyloid in dementia patients has been greatly enhanced by the use of amyloidbinding agents such as Pittsburgh compound B [17], which also demonstrated amyloid burden in DLB. An Australian study reported more variable cortical PiB binding in DLB patients than in $\mathrm{AD}$ [18], whereas a subsequent examination of $\mathrm{PiB}$ binding in LBDs including $\mathrm{DLB}, \mathrm{PDD}$, and $\mathrm{PD}$, compared with $\mathrm{AD}$ and normal patients, showed higher amyloid burden in $\mathrm{DLB}$ and $\mathrm{AD}$ than in PDD, PD, or NC patients [19]. Amyloid load was highest in LBD patients in the parietal and posterior cingulate regions, corresponding to visuospatial impairments on neuropsychological testing, suggesting that amyloid deposition could partly contribute to the clinical presentation of LBDs.

2.2. Tau Genetics and Biomarkers in LBD. Mutations in the tau gene on chromosome 17 may also present with phenotypic features of PDD or DLB, but they differ pathologically from these disorders in that LBs are generally absent [20]. Tau-bearing neurofibrillary tangles remain one of the pathological hallmarks of AD but are also central to a diverse group of disorders termed "tauopathies" which include progressive supranuclear palsy, corticobasal ganglionic degeneration, frontotemporal dementia (FTD) with parkinsonism linked to chromosome 17, and other disorders [21]. Tau is a microtubule binding protein, which acts to stabilize tubulin polymerization in microtubules critical for axonal 
TABLE 1: Genetics and biomarkers in LBD.

\begin{tabular}{|c|c|c|c|c|}
\hline & $\begin{array}{l}\text { Biochemical } \\
\text { marker }\end{array}$ & Gene defect & Relevance to LB disorders & $\begin{array}{c}\text { Source of } \\
\text { biomarker }\end{array}$ \\
\hline \multirow[t]{2}{*}{ AD lesions } & $\mathrm{A} \beta$ & $\begin{array}{l}\text { APP: K670M/N671L and so forth. } \\
\text { PS1: H163R and so forth PS2: } \\
\text { N141I and so forth }\end{array}$ & Deposited in plaques & CSF, plasma \\
\hline & Tau & $\begin{array}{l}\text { Tauopathy: P301L, N279K, K317M, } \\
\text { and so forth }\end{array}$ & $\begin{array}{l}\text { Found in NFT in AD brain, released after } \\
\text { neuronal damage }\end{array}$ & CSF \\
\hline \multirow{3}{*}{$\begin{array}{l}\mathrm{PD} / \mathrm{DLB} \\
\text { lesions }\end{array}$} & $\begin{array}{l}\alpha \text {-synuclein } \\
(\text { PARK1/4) }\end{array}$ & $\begin{array}{l}\text { A53T, A30P (PD), G209A (DLB), } \\
\text { E46K, triplication (PD \& DLB) }\end{array}$ & $\begin{array}{l}\text { Mutation } \rightarrow \uparrow \alpha \text {-syn aggregation. } \\
\text { LB component, toxic oligomers and } \\
\text { protofibrils }\end{array}$ & $\begin{array}{l}\text { CSF, skin } \\
\text { cells, platelets }\end{array}$ \\
\hline & $\beta$-synuclein & P123H, V70M (DLB) & $\begin{array}{l}\text { Inhibit } \alpha \text {-syn aggregation: mutant causes } \\
\text { degeneration }\end{array}$ & CSF \\
\hline & $\gamma$-synuclein & SNP in DLBD & $\begin{array}{l}\text { Amyloidogenic: affects neuronal and } \\
\text { axonal cytoskeleton }\end{array}$ & $\begin{array}{l}\text { Ventricular } \\
\text { CSF }\end{array}$ \\
\hline \multirow{5}{*}{$\begin{array}{l}\text { Proteostasis/ } \\
\text { oxidative } \\
\text { stress }\end{array}$} & Parkin (PARK 2) & $\begin{array}{l}\text { K161N, W453Stop, 202-203delAG, } \\
\text { M192L, K211N, and so forth }\end{array}$ & $\begin{array}{l}\text { Ubiquitin E3 ligase, LOF mutation in PD } \\
\text { alters mitophagy }\end{array}$ & ND \\
\hline & $\begin{array}{l}\text { UCHL-1 } \\
\text { (PARK 5) }\end{array}$ & I93M, S18Y (SNP) & $\begin{array}{l}\text { Neuronal deubiquitinating hydrolase; } \\
\text { impaired synaptic and cognitive function } \\
\text { in AD \& PD }\end{array}$ & ND \\
\hline & PINK 1 (PARK 6) & $\begin{array}{l}\text { A168P, A217D, E417G, E240K, and } \\
\text { so forth (PD) }\end{array}$ & $\begin{array}{l}\text { Mitochondrial serine/threonine kinase; } \\
\text { LOF mutation in PD alters mitophagy }\end{array}$ & ND \\
\hline & DJ-1 (PARK7) & $\begin{array}{l}\text { M26I, D149A, G78G, R98Q (PD), } \\
\text { L166P (PD \& DLB) }\end{array}$ & $\begin{array}{l}\text { Redox-dependent chaperone; LOF } \\
\text { mutation in PD }\end{array}$ & CSF, plasma \\
\hline & LRRK2 (PARK 8) & $\begin{array}{l}\text { G2019S, duplication, triplication } \\
\text { (PD) }\end{array}$ & $\begin{array}{l}\text { Gain of function mutant in PD?DLB: } \\
\text { interacts with } \alpha \text {-syn and tau, and with } \\
\text { parkin in apoptotic cell death }\end{array}$ & ND \\
\hline Cytoskeletal & NF & NEFM (PD) & $\begin{array}{l}\text { Disrupted NF } \rightarrow \text { abnormal axonal } \\
\text { transport; released in cell damage }\end{array}$ & CSF \\
\hline $\begin{array}{l}\text { Lysosomal } \\
\text { dysfunction }\end{array}$ & GBA & $\begin{array}{l}84 \text { dupl G, IVS } 2+1, \text { N370S, L444P } \\
\text { (PD) }\end{array}$ & $\begin{array}{l}\text { Gaucher's disease, abnormal lysosomal } \\
\text { function/autophagy in PD }\end{array}$ & CSF, plasma \\
\hline Inflammation & $\begin{array}{l}\text { IL- } 1 \alpha, \text { IL- } 1 \beta \\
\text { IL- } 6, \text { TNF } \alpha\end{array}$ & SNP: IL- $1 \beta-511$, TNF- $\alpha-308$ & $\begin{array}{l}\alpha \text {-syn-induced microglial activation } \rightarrow \uparrow \\
\text { secretion of neuroinflammatory } \\
\text { mediators }\end{array}$ & CSF \\
\hline
\end{tabular}

CSF: cerebrospinal fluid; GBA: glucocerebrosidase; A $\beta$ : $\beta$-amyloid; NF: neurofilament; ND: not yet determined; PD: Parkinson's disease; DLB: Dementia with Lewy body; UCHL1: ubiquitin carboxy terminal hydrolase L1; PINK 1: PTEN-induced putative kinase 1; LRRK2: leucine-rich repeat kinase 2; LOF: loss of function; SNP: single nucleotide polymorphism.

cytoskeletal integrity and function. In disease, tau protein truncation at Glu 391 or hyperphosphorylation causes microtubule destabilization and aggregation of unbound tau into paired helical filaments (PHFs) leading to characteristic tangle formations [22]. Unlike the tauopathies, no direct pathogenetic tau mutations have been identified in LBDs, but tau pathology appears to be a consistent feature among neurodegenerative dementias including $\mathrm{AD}$ and LBDs, and given the pathological overlap, they might share similar pathogenetic pathways (reviewed in Stoothoff and Johnson) [23]. The Ser/Thr kinase and glycogen synthase kinase$3 \beta$ (GSK3 $\beta$ ), in concert with other molecules such as fyn kinase, normally regulate tau function but with aberrant activation accelerate the hyperphosphorylation of tau in neurodegenerative disease. Similarly, the cell cycle family kinase and cyclin-dependent kinase 5 (Cdk5/p35), active during normal brain development and involved in regulatory tau phosphorylation during mitosis, may also contribute to PHF formation.
Consequently, both total tau and hyperphosphorylated forms have been widely investigated and detected in CSF, but not serum, by enzyme-linked immunosorbent assay methods. In the differentiation of dementia types, Arai et al. initially reported elevated total CSF tau levels in AD but not in $\mathrm{PD}$, but subsequently, they showed that total tau was also increased in DLB at similar levels to AD [24]. Yet, others have found differences for both total and phospho-tau (p-tau) in differentiating DLB from AD [25], and levels of total tau and p-tau 181 were significantly increased in autopsy-confirmed DLB patients [26]. In clinically diagnosed dementia cases, CSF p-tau 231 discriminated AD from non-AD dementias as a group, where levels were significantly higher in $\mathrm{AD}$ patients compared with DLB, FTD, vascular dementia, other disorders, and control subjects [27]. Separation of DLB from $\mathrm{AD}$, however, was less robust, provided that CSF p-tau 231 levels were also increased in DLB. Clinically diagnosed DLB cases also showed elevated levels of CSF p-tau 181 compared with controls [28], and Hampel et al. reported 
that $\mathrm{p}$-tau 181 provided the best discrimination of DLB from AD yielding a sensitivity of $94 \%$ and specificity of $64 \%$ [29]. In autopsy-confirmed DLB and AD patients, however, sensitivity decreased to $75 \%$ and specificity to $61 \%$, with a diagnostic accuracy reported as $73 \%$ [30].

\section{Synucleins: Genetics to Biomarkers in the Lewy Body Disorders}

\subsection{Pathogenetics of Synucleins in LBD}

3.1.1. Functions of $\alpha$-Synuclein. LBs are filamentous inclusions consisting primarily of the presynaptic protein $\alpha$ synuclein ( $\alpha$-syn), which might have several roles in vivo. Studies demonstrate that it is localized to multiple neural tissues, including high expression in neocortex and hippocampus, and that expression increases during acquisitionrelated synaptic plasticity [31]. Interaction with tubulin suggests $\alpha$-syn could be a microtubule-associated protein similar to tau $[32,33]$, and it is highly active in various membrane lipid bilayers such as in presynaptic vesicles acting as a chaperone for soluble NSF attachment protein receptor (SNARE) complex formation [34], in neuronal Golgi apparatus influencing protein trafficking [35] and in the inner membrane of neuronal mitochondrial [36]. The synucleins might act to preserve membrane stability, provide antioxidant function, and assist with membrane turnover, although the actual role of synucleins remains elusive $[37,38]$. Because of its association with LB and the tendency to self-aggregate into pathological oligomers and ultimately fibrillar structures [39], $\alpha$-syn plays a central role in the pathogenesis of LBD, hence the alternate designation "synucleinopathies." The degree of $\alpha$-syn immunoreactivity in cortical LBs correlates with cognitive severity and disease progression in PDD and DLB $[4,40]$. Also, the protein can be recovered from filaments in purified Lewy bodies from PDD and DLB brain [41], and recombinant $\alpha$-syn tends to form Lewy body-like fibrillar structures in vitro [42].

3.1.2. $\alpha$-Synuclein Mutations in PD and DLB. In the past decade, tremendous advances have been made in understanding the genetic factors influencing the pathogenesis of Lewy body disorders. Compelling evidence for a genetic basis for PD and DLB followed the discovery of mutations in the $\alpha$-syn gene (PARK1/4) in patients with autosomal dominant familial Parkinson's disease, and subsequently, mutations were identified in patients with both sporadic and familial DLBs. From a susceptibility marker on chromosome 4q2123 that segregated with the PD phenotype in Italian and Greek kindreds, A53T [43] and A30P [44] were the first two missense mutations in $\alpha$-syn associated with familial Parkinson's disease. Clinical analysis of the Italian A53T mutation revealed phenotypic variability over the disease course with several individuals demonstrating moderate to severe dementia [45]. Subsequently, a case of clinically and pathologically well-characterized DLBD in the United States and a Greek proband of DLB with a family history of PD were both determined to have the A53T $\alpha$-syn mutation [46, 47].
Another mutation, E46K, was discovered in a Spanish family presenting with autosomal dominant DLB [48], and in genetic studies of a large family with the spectrum of Lewy body phenotype ranging from PD to DLB, $\alpha$-syn gene triplication was described, causing $\alpha$-syn overproduction similar to the trisomy effect observed in Down syndrome patients [49].

Autosomal dominant point mutations are shown to affect the aggregative properties of $\alpha$-syn, which has mechanistic implications for the pathogenesis of LBD. Compared to wildtype $\alpha$-syn, biophysical analyses reveal that $\alpha$-syn aggregation is folding state dependent, where A53T and A30P mutated proteins cause increased aggregation only from the partially folded intermediate state and not the monomeric state [50]. A53T $\alpha$-syn transgenic mice have increased oligomerization of the protein in brain regions devoid of inclusions as well as those areas with more abundant lesions and neurodegeneration, and consistent with prior biophysical findings, $\alpha$-syn toxicity in these mice was dependent on the conformation of intermediate species [51]. In fact, the E46K mutation, as well as the others not only increase the tendency toward aggregation, but also promote formation of annular protofibrillar structures, causes pore formation in various membranes and neuronal damage [52].

3.1.3. $\beta$-Synuclein Mutations in DLB. $\alpha$-Syn is a member of a larger family of synuclein proteins which also includes $\beta$ synuclein ( $\beta$-syn) and $\gamma$-synuclein ( $\gamma$-syn). $\beta$-syn has recently been implicated in PD and DLB pathogenesis, but its precise role in disease is still emerging. Despite having strong homology with $\alpha$-syn, it is not clearly amyloidogenic, but is highly localized to presynaptic sites in neocortex, hippocampus, and thalamus like $\alpha$-syn $[53,54]$. Normal $\beta$-syn may act as a biological negative regulator of $\alpha$-syn. In bigenic $\alpha$ syn/ $\beta$-syn-overexpressing mice and in doubly transfected cultured cells, $\beta$-syn ameliorated amyloidogenicity, neurodegenerative changes, and motor deficits induced by $\alpha$-syn overexpression alone [55]. On the other hand, mutated $\beta$ syn leads to neuronal damage and disease and augments neurodegeneration, perhaps through a loss of its natural regulator function. Two novel $\beta$-syn point mutations, $\mathrm{P} 123 \mathrm{H}$ and V70M, were found in highly conserved regions of the $\beta$ syn gene in respective familial $(\mathrm{P} 123 \mathrm{H})$ and sporadic (V70M) DLB index cases [56], where abundant LB pathology and $\alpha$-syn aggregation was present without $\beta$-syn aggregation. $\mathrm{P} 123 \mathrm{H} \beta$-syn overexpression in transgenic mice resulted in axonal damage, gliosis, profound memory, and behavioral deficits [57]. These phenomena may involve $\alpha$-syn, since bigenic mice overexpressing $\alpha$-syn with $\mathrm{P} 123 \mathrm{H} \beta$-syn show greater deficits compared with monogenic mice and compared with $\mathrm{P} 123 \mathrm{H} \beta$-syn expressed with $\alpha$-syn knockout, implying that the $\mathrm{P} 123 \mathrm{H}$ mutation has a synergistic effect with other synucleinopathies to cause neurodegeneration. $\mathrm{P} 123 \mathrm{H}$ as well as V70M $\beta$-syn mutations might also injure neurons by disrupting normal lysosomal pathways and corresponding cellular autophagic processes [58].

3.1.4. Association of $\gamma$-Synuclein with LBD. Unlike the other synuclein family members, $\gamma$-syn or persyn is largely 
expressed in the cell bodies and axons of primary sensory neurons, sympathetic neurons, and motor neurons as well as in brain [59]. In cancer biology, $\gamma$-syn is associated with abnormally altering cellular mitotic checkpoints in various types of malignancies, making them more aggressively metastatic [60], but as far as neurodegeneration, it is the most recent synuclein member to be linked to LBD neuropathology and the least well understood. Single-nucleotide polymorphisms in all three synucleins have been associated with sporadic DLBD, most prominently $\gamma$-syn [61], and in sporadic PD, DLB, and LBV patients, $\gamma$-syn antibodies, as well as $\beta$-syn and $\alpha$-syn reveal unique hippocampal axonal pathology [62]. In vivo, $\gamma$-syn overexpression in transgenic mice shows age- and dose-dependent neuronal loss throughout the neuraxis, especially in spinal motor neurons, where $\gamma$-syn-bearing inclusions, gliosis, and alterations in heat shock protein and neurofilament structure are found [63], perhaps suggesting relevance to motor neuron disease associated with dementia. In vitro evidence further supports a cytoskeletal role for $\gamma$-syn in maintaining neurofilament structure; $\gamma$-syn overexpression in cultured neurons causes disruption of the neurofilament network by destabilizing the structural integrity of neurofilament- $\mathrm{H}$ allowing degradation by calcium-dependent proteases, which has implications for neurodegeneration [64].

\subsection{Synucleins as Biomarkers of $L B D$}

3.2.1. Synucleins in the Extracellular Compartment. Synucleins are known as intracellular molecules, but they also appear in extracellular and peripheral fluids from active and passive processes. Evidence suggests that turnover and secretion of these proteins might occur during normal cellular processing, releasing synucleins into extracellular space and hence into peripheral sites. In transfected and untransfected cultured neuroblastoma cells, $15 \mathrm{kDa} \alpha$-syn is released into surrounding media [65], and furthermore, not only monomeric $\alpha$-syn but also aggregated forms are secreted in an unconventional exocytic manner into extracellular fluid in response to proteasomal and mitochondrial dysfunction [66]. Remarkably, Desplats et al. recently showed that neuronally secreted $\alpha$-syn can also be taken in endocytically by other neurons or glia as a means of transmitting pathology [67]. Secreted $\alpha$-syn interacts with various molecules such enzymes; in cultures, matrix metalloproteinase- 3 cleaves native $\alpha$-syn to smaller proteolytic fragments that enhance its aggregative properties [68]. Whether $\beta$-syn and $\gamma$-syn also undergo unconventional exocytosis and secretion remains unknown, but given structural and functional similarity to $\alpha$-syn, the possibility exists. Certainly, synaptic and axonal damage reflecting neurodegeneration may also allow release of synucleins into the extracellular millieu and access to peripheral fluids such as CSF and blood.

3.2.2. $\alpha$-Synuclein as a PD and DLB Biomarker. Multiple forms of $\alpha$-syn are released into cerebrospinal fluid (CSF) and other biological fluids. Full-length $\alpha$-syn has been recovered from lumbar CSF from living normal control,
PD and DLB patients $[69,70]$, and also from postmortem CSF from DLB and other neurodegenerative diseases [71]. Comparative findings regarding differences in CSF $\alpha$-syn levels among various neurodegenerative diseases, however, are difficult to interpret because of inconsistent observations. In $\mathrm{PD}$, a smaller early study showed that no differences in full-length CSF $19 \mathrm{kDa} \alpha$-syn have been found in relation to control individuals [69], but a recent effort using a new Luminex assay in a larger sample controlling for extraneous influences showed significantly decreased levels in $\mathrm{PD}$ compared to controls with $92 \%$ disease sensitivity and $58 \%$ specificity [72]. Elevated $\alpha$-syn levels, however, were found in $\mathrm{DLB}, \mathrm{AD}$, and vascular dementia with no differences among them [71]. Perhaps more intriguing, higher-molecular weight aggregated $\alpha$-syn species in CSF might be associated with PD and DLB. Reduced levels of a $24 \mathrm{kD} \alpha$-syn-immunoreactive band were found in DLB CSF and correlated directly with declining cognition [73]. Moreover, using a specific enzyme-linked immunosorbent assay (ELISA), soluble aggregated $\alpha$-syn oligomers in CSF were significantly increased in PD patients compared against control subjects, $\mathrm{AD}$ and progressive supranuclear palsy, and specificity ranged from approximately 85 to $87 \%$, while sensitivity was about $53-75 \%$ range [74].

Plasma $\alpha$-syn detected by immunoblotting was decreased in PD compared with age-matched control subjects, and those PD patients with age-at-onset prior to 55 years (earlyonset) had significantly lower levels than those with onset after 55 years of age (late-onset) [75]. In addition, soluble oligomeric $\alpha$-syn detected by specific ELISA was significantly elevated in plasma from PD. This test demonstrated a specificity of approximately $85 \%$, a sensitivity of $53 \%$, and a positive predictive value of 0.818 [76]. Although measurement of plasma $\alpha$-syn appears interesting as a biomarker, it was reported that skin cells and platelets are also sources for $\alpha$-syn, and their levels did not correlate with disease presence or severity [77]. Moreover, red blood cells are also a major source of $\alpha$-syn [78], and thus, plasma could be contaminated by $\alpha$-syn not originating from brain, which might render interpretation of results difficult. One promising consideration for the future exploration of $\alpha$-syn as an LBD biomarker will be the development of novel imaging compounds and techniques, similar to amyloid imaging, to specifically target and visualize $\alpha$-syn distribution in the PD and LBD brain. The availability of such methods will be a significant advance in biomarkers for synucleinopathies.

3.2.3. $\beta$-Syn and $\gamma$-Syn as Potential Biomarkers in Lewy Body Disorders. Due to their increasing importance in LBD pathogenesis, $\beta$-syn and $\gamma$-syn, as much as $\alpha$-syn, might be excellent targets as peripheral markers of disease. As such, levels of these synucleins might be altered in the CSF of patients with $\mathrm{PD} / \mathrm{PDD}$ and DLB, reflecting the underlying degenerative processes in brain. No studies to date have examined $\beta$-syn levels in peripheral fluids in relation to neurodegenerative disease, but a small study reported elevated postmortem ventricular CSF $\gamma$-syn levels 
in $\mathrm{DLB}, \mathrm{AD}$, and vascular dementia patients, with the highest levels seen in DLB patients [71]. More detailed examination of both $\beta$-syn and $\gamma$-syn as a peripheral disease markers in well-characterized populations of PD, DLB, and other disorders is warranted to determine their specificity and sensitivity in the synucleinopathies.

\section{DJ-1 in the Lewy Body Disorders}

4.1. Functional Role of DJ-1 in Lewy Body Diseases. Recently, DJ-1 (PARK 7) has emerged as a significant molecular target of interest in LBD principally because of its genetic association with PD and its increasing importance in cellular oxidative neuroprotection. Although its exact role is unknown, multiple functions have been assigned to the DJ-1 protein. Described by Nagakubo et al. as a mitogen-dependent oncogene involved in Ras-related signaling pathways [79], it shares structural homology with the carboxy-terminal domain of Escherichia coli HPII catalase and is reported to possess catalase activity which reduces oxidative stress in cultured cells [80]. It also binds to and regulates the PIAS SUMO-1 ligase and is itself posttranslationally modified by sumoylation $[81,82]$. Of relevance to Lewy body formation and neurotoxicity, DJ-1 displays redox-dependent chaperone activity conferring proper protein folding and thermal stability, which in fact, also inhibits $\alpha$-syn aggregation [80]. The overexpression of DJ-1 in rats protects nigral dopaminergic neurons against degeneration involving 6-hydroxydopamine, while mutant DJ-1 in mice causes abnormal dopamine reuptake and susceptibility to 1-methyl- 4-phenyl-1,2,3,6tetrahydropyridine (MPTP) toxicity [83]. Deletion of DJ1 homologs in Drosophila renders them sensitive to $\mathrm{H}_{2} \mathrm{O}_{2}$, paraquat, and rotenone toxicity [84].

4.2. DJ-1 Mutations and Possible Relevance to LBD. No less than 13 gene mutations have been identified in DJ-1 in atypical younger-onset PD patients, but their significance to idiopathic late-onset PD remains uncertain. In autosomal recessive early-onset $\mathrm{PD}$ from consanguineous families, a complete DJ-1 deletion in a Dutch family and a point mutation L166P in an Italian case were identified [85]. When expressed in cultured cells, L166P appears to be a loss-of-function mutation which leads to DJ-1 functional instability, degradation by the proteasome system [86, 87], abnormal translocation of DJ-1 to mitochondria, and loss of chaperone activity [80]. The importance of DJ-1 gene alterations in dementia and DLB, however, is uncertain. One report found no impact on dementia risk of the DJ-1 $14 \mathrm{~kb}$ deletion [88], and analysis of an insertion/deletion variant (g.168_185del) in DJ-1 in a larger sample of patients also showed no association with either PD or DLB compared to control patients [89]. Given these early negative findings, the relevance of DJ-1 genetic mutations to DLB and other LBD is not known. At present, no patient harboring a DJ-1 mutation has come to autopsy, so the precise pathology is not known. Although DJ-1 mutant cases may ultimately not be LBDs, it is possible that alterations in DJ-1 may somehow influence the aggregation of $\alpha$-syn and LB formation [80] or contribute to pathogenesis by other molecular pathways.

DJ-1 is found in brain across a wide range of neurodegenerative diseases including PD, FTD, AD, DLB, and LBVAD, and demonstrates striking association with neuropil threads and neurofibrillary pathology in neocortex and subcortical brain regions in these disorders [90]. Interestingly, this association with tau pathology was seen in DLB and LBV brains, suggesting that as a chaperone molecule, DJ-1 may be involved in tangle formation, and the binding of DJ1 with these lesions could abolish the normally protective effect of DJ-1, enhancing oxidative neurotoxicity. Wang et al. observed that DJ-1 knockout mice have markedly abnormal hippocampal long-term depression accompanied by a less severe abnormality in long-term potentiation, which was reversed by the D2/3 agonist quinpirole, indicating that DJ-1 has a role in dopamine-dependent signaling in hippocampal plasticity [91]. This implies that DJ-1 may be important in the maintenance of memory and cognition.

4.3. DJ-1 as a Potential Biomarker for Lewy Body Diseases. Given its pathogenetic significance, DJ-1 could be a candidate biological marker for DLB and LB and might serve as a means of monitoring in vivo oxidative damage and protein misfolding. Although intracellular and mitochondrial in localization, DJ-1 is presumed to be secreted perhaps specifically under disease conditions which induce oxidative damage. Using semiquantitative immunoblotting, we previously identified DJ-1 in CSF of sporadic PD patients, where levels were significantly elevated compared with controls. Levels were higher in the earlier stage PD cohort (Hoehn-Yahr stages I-II) than in the more severe patients (Hoehn-Yahr stages III-IV) [92]. Similarly, plasma DJ-1 levels in PD patients were markedly increased compared to controls, but unlike CSF, levels were relatively higher in late stage (III-IV) rather than early stage PD (I-II) [93]. The reason for this difference between plasma and CSF DJ-1 is unknown, but we surmised previously that since CSF DJ-1 originates from a central source produced mainly by reactive glia, early increases in CSF DJ-1 levels probably represent an early protective response to damage, whereas plasma DJ-1, like other plasma disease markers, likely represents peripheral oxidative stress damage. In fact, DJ-1 is secreted into blood in breast cancer, melanoma, familial amyloid neuropathy, and stroke [94-96]. In the largest study to date, Hong et al. developed a more sensitive and quantitative Luminex assay for CSF DJ-1 to complement immunoblotting mass spectrometric and chromatographic analysis methods and found decreasing rather than increasing levels of DJ-1 in PD CSF compared with control patients [72]. The $90 \%$ disease sensitivity and 70\% disease specificity for PD using this method approaches minimal desired parameters for a clinically useful biomarker for PD. Importantly, the study highlighted the fact that DJ-1 levels are greatly influenced by such variables as the extent of blood contamination and patient age, which could account for some of the variability across studies. Of note, DJ-1 is also subject to oxidative modifications in $\mathrm{PD}$ and $\mathrm{AD}$ brain tissue, and this might be 
measured in peripheral fluids as well, as another monitor of oxidative damage [97]. CSF DJ-1 remains a promising and perhaps clinically useful biomarker for $\mathrm{PD}$, but as far as DLB and other LBD, it is unknown whether CSF levels of DJ1 are altered. Since plasma DJ-1 is increased in DLB, it is hypothesized that CSF DJ-1 may also be elevated. Further investigation will be necessary to clarify the utility of DJ-1 as a biomarker in DLB and LBD.

\section{Glucocerebrosidase as a Novel Biomarker for Lewy Body Disorders}

5.1. Glucocerebrosidase Mutations Influence PD and DLB. Many clinicopathologic parallels can be drawn between the lysosomal storage disorders, such as Niemann-Pick, Sandhoff's, Tay-Sachs disease and others, and the age-related neurodegenerative disorders, when considering the aberrant accumulation of pathological substances (e.g., lysosomal sphingomyelin in Niemann-Pick disease versus synucleins in PD and DLB) and the phenotypes of neuronal loss and cognitive deterioration found in both. Common to these diseases are abnormalities in lysosomal and autophagic mechanisms as part of a larger disruption of cellular proteostasis leading to abnormal storage/accumulation of toxic materials and neuronal damage. In the past few years, an altogether unexpected pathogenetic relationship emerged between Gaucher's disease (GD), a prototypic storage disease, and the synucleinopathies. Despite its overall rarity, GD is the most common inherited lysosomal storage disease, especially in the Ashkenazi Jewish population. It is caused by autosomal recessive gene mutations in the glucocerebrosidase (GBA) gene (chromosome 1q21), leading to either partial or complete deficiency of GBA, and hence, toxic lysosomal accumulation of its substrate, glucosylceramide, in multiple cell types including neurons [98]. Recent reports documented an increased incidence of PD in heterozygous relatives of patients with GD $[99,100]$, but interest in this phenomenon was propelled by the finding that GBA mutations were in fact more common in $\mathrm{PD}$ patients of Ashkenazi background compared with $\mathrm{AD}$ patients and $\mathrm{PD}$ patients in the general population [101-103]. Moreover, more severe GBA mutations such as 84 dupl G and IVS $2+1$ were associated with a greater degree of PD risk, compared with less severe GBA mutations such as N370S [104]. The relationship between PD and GBA has now been replicated in much larger international studies with the most common mutations being L444P and N370S, and about 28 GBA mutations are presently recognized [105].

Interestingly, in a study of British patients with PD and GBA mutations, all 17 carrier patients demonstrated abundant $\alpha$-syn neuropathology with Braak stage 5-6 severity and common neocortical LB pathology. Clinically, these patients had earlier age at onset, and hallucinations were present in $45 \%$ of patients, while $48 \%$ had cognitive impairment or dementia consistent with PDD [106]. Greater severity of GBA mutation also predicted the presence of cognitive impairment in PD patients; $56 \%$ of severe GBA mutation carriers had cognitive impairment compared to $25 \%$ of mild mutation carriers [107]. These observations suggest a much broader link between GBA mutations and the dementia phenotype of LBD. In fact, examination of GBA gene alterations in DLB patients, with and without concomitant LBV-type AD pathology, showed that the majority of GBA mutations were found in DLB patients rather than in $\mathrm{PD}$, with a mutation rate in DLB ranging from 18 to $23 \%$ overall [108, 109]. The proportion of DLB patients with GBA mutations was higher in those with pure neocortical LB pathology compared to those with mixed $\mathrm{LB}$ and $\mathrm{AD}$ pathology and to those with predominantly brainstem LB. A significant association was also found between GBA mutation status and the presence of LB, indicating that altered GBA might play a role in their formation and in synucleinopathy [108].

\subsection{Glucocerebrosidase and Chaperone-Mediated Autophagy} in LBD. Important in neurodegeneration, disrupted cellular proteostasis represents a state in which an imbalance exists between effective functioning of the innate cytoprotective machinery and excessive accumulation and aggregation of abnormally misfolded proteins, leading to neurotoxicity. It is increasingly apparent that chaperone-mediated autophagy (CMA) and lysosomal degradation pathways are important in maintaining cellular proteostasis as part of a larger network of cellular actions, with particular relevance for neurodegenerative diseases. Recently, as evidence for CMA dysfunction in synucleinopathies, a significant decrease in autophagy markers was reported in substantia nigra from PD brain [110]. Soluble forms of $\alpha$-syn, including monomers, oligomers, and even protofibrils, are normally cleared through the CMA/lysosomal degradation by interacting with the chaperone, heat shock cognate-70, and becoming internalized into lysosomes via the Lamp-2a membrane receptor $[111,112]$. Studies have indicated that $\alpha$-syn shares a common pentapeptide structure with other lysosomal substrates, designating it as a target for removal by this pathway [111], and the lysosomal structure is critical to maintaining the internal acidic environment, allowing lysosomal hydrolases to degrade $\alpha$-syn into peptides released into the cytosol [112]. Mutant GBA could therefore disrupt lysosomal activity leading to abnormal accumulation of nondegraded $\alpha$-syn, which then aggregates to toxic soluble oligomers and protofibrils. Also, abnormalities in the ubiquitin-proteasome system (UPS) are present in AD and PD, and GBA alterations might secondarily overwhelm the ability of UPS to remove accumulated $\alpha$-syn, promoting aggregation and neurotoxicity [113]. Pathologically, in GD with parkinsonism, $\alpha$-syn-positive inclusions were observed in neurons in hippocampal CA2-4 regions, while cortical synuclein pathology was identified in other GD cases [114]. Further, parkin, an E3 ubiquitin ligase also implicated in $\mathrm{PD}$, has been shown to affect the stability of mutant GBA and increase its degradation causing further lysosomal dysfunction [115].

5.3. Glucocerebrosidase as a LBD Biomarker. Because of the importance of mutant GBA function to PD and DLB 
pathogenesis, the issue arises as to whether the measurement of GBA activity, or a perhaps other related molecules, might be utilized as a biological marker. The activity of peripherally secreted GBA was measured in plasma and CSF in a 10-month-old female with GD with the aim of monitoring the effect of experimental Cerezyme replacement therapy [116]. Baseline GBA activity was detected in both plasma $\left(2.7 \times 10^{-6} \mathrm{U} / \mu \mathrm{L}\right)$ and $\operatorname{CSF}\left(0.096 \times 10^{-6} \mathrm{U} / \mu \mathrm{L}\right)$, although CSF activity was several magnitudes lower than plasma. Intravenous Cerezyme, a macrophage-targeted GBA, rapidly raised the plasma activity within 1 hour and CSF activity by 2.3 -fold at 3 hours, both returning to baseline after 24 hours. This study suggests the intriguing possibility that GBA activity, especially in CSF and plasma, might be useful in monitoring the efficacy of novel therapies involving CMA and lysosomal function. To extend this observation, Balducci et al. determined that multiple lysosomal hydrolases, including GBA, are significantly decreased in the lumbar CSF of PD patients [117], perhaps supporting a more widespread lysosomal dysfunction in PD not limited to GBA alone. In this regard, other lysosomal enzymes such as mannosidase and $\beta$-hexosaminidase might be important additional biomarker targets for neurodegeneration. Moreover, in DLB, AD, and FTD patients, lysosomal enzyme activities in CSF demonstrated a very specific pattern of decrease, in which only DLB showed significant decreases in CSF activity of $\alpha$-mannosidase, $\beta$-mannosidase, GBA, galactosidase, and $\beta$-hexosaminidase, whereas in $\mathrm{AD}$ and FTD, only CSF $\alpha$-mannosidase activity was significantly diminished [118]. In DLB, CSF GBA activity showed the greatest magnitude of decrease, reinforcing its importance in the LBD, but also noteworthy is the fact that $A D$ and FTD showed decreased $\alpha$-mannosidase activity, suggesting that this might be another important factor in lysosomal dysfunction in neurodegeneration. Indeed, these promising candidates need to be investigated further to establish diagnostic accuracy in terms of disease specificity and sensitivity in cohorts of PD, DLB, and other dementing disorders.

\section{Miscellanous Candidate Biomarkers}

6.1. Inflammatory Cytokines. Polymorphisms in proinflammatory cytokine genes including IL- $1 \alpha$, IL- $1 \beta$, and TNF$\alpha$ are associated with increased risk in AD [119]. In PD, several case control genetic analyses have demonstrated that homozygous carriers of the IL- $1 \beta-511$ and TNF- $\alpha-308$ promoter region variants have increased disease risk [120, $121]$, and that earlier age at onset in PD was associated with IL- $1 \beta-511$ homozygosity at allele 1 [122]. But as yet, no such genetic alterations in cytokines genes have been reported in DLB. Similar to A $\beta$-induced upregulation of inflammatory cytokines in $\mathrm{AD}$, soluble secreted $\alpha$-syn in the extracellular space in LBD might also induce the production of a variety of neuroinflammatory mediators into the extracellular fluid. For instance, microglial activation in response to stimulation by secreted $\alpha$-syn from cultured cells and from overexpression in transgenic mouse models occurs in a dose-dependent manner, causing release TNF- $\alpha$, IL- $1 \beta$, and IL-6 [123]. Because secreted CNS cytokines are readily detected in CSF, they have been extensively examined as potential disease biomarkers. IL-1 $\beta$, IL-2, IL-6, and TNF$\alpha$ are all upregulated in PD brain, as well as in CSF from PD patients [124-126], and Chen et al. showed that plasma IL-6, but not IL- $1 \beta$, TNF- $\alpha$, or other acute phase reactants, predicted risk for future PD in males [127]. In terms of DLB, CSF IL- $1 \beta$ levels, which were relatively low, did not differ compared to AD or normal controls and could not distinguish them apart. Comparable increases in CSF IL-6 levels were found in AD and DLB, but again not significantly different from each other to be of diagnostic value [128]. Indeed, the neuroinflammatory cytokines may be important as a pathogenetic response to CNS injury caused by accumulation of amyloidogenic proteins, but their role as biomarkers for the LBD, especially for DLB, is still unclear.

6.2. Neurofilament Proteins. Disorganization and breakdown in the cytoskeletal network occurs in various LBDs and other neurodegenerative diseases, and as discussed, gammasynuclein and proteolytic degradation of the cytoskeleton may be involved. As a result, a failure of normal axonal transport results from the accumulation of disrupted neurofilament molecules within the neuropil, causing neuronal demise [129]. Recently, a mutation in the NEFM gene encoding the rod domain $2 \mathrm{~B}$ of neurofilament $\mathrm{M}$ (NF-M) which causes aberrant NF assembly was identified in a single earlyonset PD patient [130]. It is recognized that in addition to $\alpha$-syn, three types of NF protein also comprise the structure of Lewy bodies [131]. Upon cell death or axonal damage, accumulated neurofilament leaks into the extracellular space, subsequently appearing in CSF and perhaps other peripheral fluids. Elevated CSF NF protein was reported in MSA and PSP, but not in PD, and this was suggested to clinically aid in differentiating parkinsonian syndromes [132]. CSF NF protein was also measured in dementia, and although increased levels were observed in DLB, late-onset $\mathrm{AD}$, and FTD, there were no differences among them [28]. Therefore, because cytoskeletal abnormalities are present in many neurodegenerative dementias as well as in PD, NF protein may be more a reflection of nonspecific alterations in neuronal and axonal function, which does not appear to able to clinically separate DLB from other disorders.

\subsection{Brain Neurotransmitter Alterations in CSF and by Imaging} Modalities. Severe cortical cholinergic deficits originating from deficiencies in the nucleus basalis of Meynert are characteristic of $\mathrm{AD}$ brain, but studies have shown that cholinergic deficits are perhaps more severe in DLB brain [5]. This suggests that measurement of cholinergic activity and/or acetylcholine (ACh) might be developed into a potential biomarker for the LBDs. Indeed, early attempts to quantify ACh or its major metabolite, choline, have shown baseline levels to be low and perhaps difficult to measure accurately. In AD, CSF ACh was reported to be significantly lower than control levels [133], while in PD and Huntington's disease patients, despite some cholinergic deficit, lumbar CSF 
ACh and choline levels did not differ from normal [134]. No studies have directly examined CSF cholinergic levels in DLB or LBDs, but recently, Shimada and colleagues employed positron emission tomography (PET) mapping of brain ACh activity in DLB and PDD patients and normal controls and demonstrated a marked reduction in cholinergic activity in medial occipital cortex of DLB and PDD, greater than that observed in PD patients without dementia [135]. Some correlation of mapped cholinergic activity with cognitive decline measured by the Mini-Mental State Exam was also found. Although preliminary, this has potential to be a more practical and sensitive cholinergic biomarker for LBD.

Because of similar nigrostriatal loss to $\mathrm{PD}$, a relative dopaminergic deficiency also exists in DLB and LBDs. CSF dopamine (DA) and its metabolites have been investigated previously in PD, and recently, Lunardi et al. showed differences in CSF DA and its metabolites, homovanillic acid (HVA) and dihydroxyphenylacetic acid (DOPAC), in PD patients, demonstrating early-stage dopaminergic loss and a correlation with the development of dyskinesia [136]. In DLB, HVA levels were significantly reduced compared with $\mathrm{AD}$, separating the disorders [137]. Similar to cholinergic activity, imaging modalities may also contribute to the assessment of dopaminergic function in the LBDs. In a small study, striatal DA uptake as measured by ${ }^{18} \mathrm{~F}$ fluorodopa PET was decreased in both caudate and putamen in DLB as compared with AD patients and controls [138]. Also, DA transporter loss was determined across multiple studies using ${ }^{123} \mathrm{I}$ - $2 \beta$-carbometoxy- $3 \beta$-(4-iodophenyl)$\mathrm{N}$-(3-fluoropropyl) nortropane ligand with single-photon emission computed tomography $\left({ }^{123} \mathrm{I}-\mathrm{FP}\right.$ CIT SPECT) and demonstrated significant loss of caudate and putaminal DA transport compared with AD and control levels [139141]. A larger phase III, multicenter study of ${ }^{123}$ I-FP CIT SPECT in possible and probable DLB patients and non-DLB comparators (mostly AD) demonstrated a mean sensitivity of $77.7 \%$ for detecting clinically probable DLB, with a specificity of $90.4 \%$ and $85.7 \%$ overall diagnostic accuracy [141]. ${ }^{123}$ I-FP CIT SPECT DA transporter imaging greatly enhanced diagnostic accuracy for DLB over clinical diagnosis alone when coupled with autopsy confirmation, raising sensitivity for DLB from $75 \%$ to $88 \%$ and specificity from $42 \%$ to $100 \%$ [139]. Furthermore, DA transporter loss in the caudate may also be inversely associated with depression, apathy, and delusions in DLB patients [142].

\subsection{Miscellaneous Imaging Biomarkers in LBD}

6.4.1. MIBG Scintigraphy as a DLB Biomarker. Autonomic failure is a common clinical finding in LBD, including PD and DLB, but not in non-LBD dementias, and therefore it has been investigated as an alternative biomarker for the diagnostic separation of DLB from other dementias. Abnormal autonomic function can be determined using cardiac ${ }^{123}$ I-meta-iodobenzyl guanidine ( $\left.{ }^{123} \mathrm{I}-\mathrm{MIBG}\right)$ imaging, a technique which assesses cardiac sympathetic nerve function in both cardiac and neurological disorders by measuring the uptake of ${ }^{123}$ I-MIBG, a norepinephrine analogue [143].
In the last decade, a series of Japanese studies consistently demonstrated delayed heart to mediastinum ratio $(\mathrm{H} / \mathrm{M})$ of ${ }^{123} \mathrm{I}$-MIBG uptake in DLB compared with AD and controls [143-146]. ${ }^{123}$ I-MIBG scintigraphy was found superior to brain perfusion SPECT imaging [147]. Estorch et al. further showed that in dementia patients followed for four years before "final diagnosis," ${ }^{123}$ I-MIBG imaging distinguished DLB from other dementias with a sensitivity of $94 \%$, specificity of 96\%, and a diagnostic accuracy of 95\% [148]. Finally, consistent with autonomic dysfunction in DLB, both early and delayed H/M ${ }^{123}$ I-MIBG uptake were significantly associated with the presence of orthostatic hypotension in DLB patients and discriminated DLB from AD even in the absence of parkinsonism [149].

6.4.2. Other Structural and Functional Imaging Biomarkers. Various magnetic resonance (MR) imaging modalities have been explored in DLB and PDD, including volumetric imaging, diffusion tensor imaging, and proton magnetic resonance spectroscopy (reviewed in Watson et al.) [150], and although not directly useful as biomarkers at present, they have revealed insights in the pathobiology of LBDs. Using conventional MRI techniques such as voxel-based morphometry and region of interest analysis, some degree of diffusion or focal frontal and parietal atrophy has been observed [151]. Atrophy has been rated at $1.4 \%$ per year in DLB brain [152], $1.31 \%$ per year in PDD, and $0.31 \%$ per year in PD [153]. Not surprising is the fact that unlike AD brain, medial temporal structures are relatively preserved in DLB and PDD, with global hippocampal loss at about $10-20 \%$ compared with controls and about $21-25 \%$ in $\mathrm{AD}$ [154]. Diffusion tensor imaging, an MR technique mapping brain microdiffusion of water in the direction of white matter tracts, has shown decreased fractional anisotropy of water movement in DLB in the precuneus and posterior cingulate areas, perhaps highlighting their role in DLB pathogenesis [155].

Brain perfusion SPECT ( ${ }^{99} \mathrm{~m}$ Tc-HMPAO SPECT) has been evaluated in its ability to diagnostically separate DLB from $\mathrm{AD}$, and in $\mathrm{AD}$, reduced relative cerebral blood flow $(\mathrm{rCBF})$ in the frontal, and medial temporal regions is characteristic, whereas in DLB, occipital hypoperfusion is often observed [156]. Colloby et al. applied statistical parametric mapping to SPECT imaging of DLB patients, more precisely showing large perfusion deficits in the left medial occipital gyrus and the bilateral central, inferior parietal, precuneate, superior frontal and cingulate regions on the brain, which are functionally consistent with frontalexecutive and visuospatial deficits in DLB [157]. Across studies, sensitivity ranged from 65 to $85 \%$ and specificity from $85-87 \%$, which appears less robust as a potential imaging marker compared with other methods.

6.5. Other PD Genes and Their Protein Products as Possible DLB Markers. Aside from $\alpha$-syn and DJ-1, numerous other mutations have been associated with familial early-onset PD and possibly LBD (Table 1). Among these gene products 
are parkin (PARK 2), UCHL-1 (PARK 5), PINK1 (PTENinduced putative kinase 1; PARK 6), and LRRK2/dardarin (PARK 8) [158]. Indeed, none of these mutations have yet been associated with prototypic LBD pathology, and it remains to be determined whether they actually represent LBDs or separate diseases with parkinsonian phenotype. Furthermore, no studies have addressed their role as biological markers of disease, but since both synucleins and DJ-1 are detected in CSF and peripheral fluids, it seems plausible that the protein products of other dominant genes in PD could be peripheral biomarker candidates for DLB and other LBD. Parkin, UCHL-1, and PINK1 genes, like DJ-1, all encode proteins important in neuroprotection in terms of maintaining protein homeostasis and preventing stress-related cellular damage, and mutations in these genes cause a loss of these critical functions. Leucine-rich repeat kinase 2 (LRRK2/dardarin), on the contrary, is linked with autosomal-dominant late-onset $\mathrm{PD}$, and mutations result in a toxic gain of function.

LRRK2/dardarin is a kinase consisting of multiple functional domains, and recent evidence suggests that physiologically, its principal function may be to regulate neurite outgrowth. Expression in cultured neurons of several LRRK2 mutations associated with familial PD, such as G2019S, increased kinase activity and significantly reduced neurite outgrowth, whereas expression of a dominant-negative mutation, K1906M, markedly increased neurite length [159]. $\mathrm{PD}$-associated mutations also generated tau-positive axonal inclusions in cultured neurons, suggesting that LRRK2 may be linked to abnormalities in tau. Indeed, expression of mutant G2019S LRRK2 in Drosophila caused activation of the Drosophila GSK-3 $\beta$ homolog and promoted tau hyperphosphorylation leading to microtubule fragmentation and dendritic pathology [160]. Similar tau hyperphosphorylation was also present in transgenic mice expressing G2019S LRRK2, and expression of both wild-type human LRRK2 and G2019S mutant LRRK2 caused abnormal dopaminergic transmission [161]. LRRK2 may also interact with $\alpha$-syn, another dominantly inherited PD gene, to exert its effect. Lin et al. showed that overexpression of LRRK2 with A53T mutant $\alpha$-syn in transgenic mice worsened neurodegeneration, while ablation of LRRK2 expression suppressed $\alpha$-syn aggregation and pathology [162], and $\alpha$-syn also activates GSK-3 $\beta$ in mice causing tau hyperphosphorylation [163], indicating that LRRK2, $\alpha$-syn, and tau alterations may all be linked in the same pathway, perhaps with LRRK2 upstream of these events. Although early, evidence has indicated that LRRK2 is also a component of LB in PD and DLB brains [164], and that LRRK2 and $\alpha$-syn interact in DLB brain and coimmunoprecipitate in cultured cells after oxidative stress challenge [165], suggesting that the LRRK2 may also be important in DLB pathogenesis. Interestingly, genome-wide association studies (GWASs) in a European cohort demonstrated that LRRK2, $\alpha$-syn, and tau are loci associated with PD risk [166], but examination of tau in a Japanese GWAS cohort failed to identify it as a PD risk locus [167], showing a population difference with regard to this locus. Certainly, population differences might apply to all risk loci examined for PD and LBD, and it is important to determine whether the relationship among LRRK2, $\alpha$-syn, and tau in PD, DLB, and other LBD is also influenced by population differences. These findings make LRRK2/dardarin an attractive candidate for examination as a potential biomarker, and if identified in CSF or peripheral fluids, they might be used with $\alpha$-syn and tau as combined biomarkers.

Furthermore, emerging evidence is redefining the roles of PINK1 and parkin in PD pathogenesis. Because energy generation is critical for cellular function, mammalian cells are highly dependent on mitochondria [168]. Depolarization and morphological defects characterize damaged or impaired mitochondria which are targeted for removal through mitophagy, a highly specialized form of autophagy in which parkin and PINK1 play a crucial role (reviewed by Vives-Bauza and Przedborski) [169]. In this process, PINK1 cleavage is inhibited by the loss of mitochondrial membrane potential, causing its lengthening and the recruitment of cytosolic parkin $[170,171]$. Voltage-dependent anion channel 1 and other outer mitochondrial membrane proteins are then ubiquitinated in a parkin-dependent manner, and this in turn recruits the binding of adapter proteins such as p62 and histone deacetylase 6 to initiate autophagosome assembly around the damaged mitochondrion and subsequent removal [169]. Of relevance to PD, mutant PINK1 and mutant parkin both cause motor dysfunction, dopaminergic loss, and abnormal mitochondrial morphology in Drosophila [172]. In this paradigm, loss of function PINK1 mutants are rescued by concurrent overexpression with wild-type parkin but not vice versa, indicating that parkin specifically acts downstream of PINK1. Also, parkin mutations have been shown to interfere with ubiquitination and the downstream steps in normal mitophagy [173]. Thus, $\mathrm{PD}$, and possibly related dementias, might be a result, to some extent, of defective mitophagy due to loss of function in PINK1 and parkin such as found in autosomal dominant early-onset PD.

Although LRRK2, parkin, PINK1, and UCHL-1 have not yet been identified in peripheral fluids, PINK1 and parkin may be a promising candidates. Unexpectedly, both PINK1 and parkin, which are normally cytosolic or targeted to mitochondria, were localized extracellularly in $\mathrm{AD}$ and multiple sclerosis brain, and colocalized with amyloid plaques, reactive astrocytes, as well as amyloid-affected vessels $[174,175]$. This suggests that both PINK1 and parkin are actively released from neurons and glia in response to injury and might be upregulated in CSF and peripheral fluids during neurodegeneration. Interestingly, given a role in mitophagy, they might also be a CSF or peripheral reflection of mitochondrial health and turnover. It remains to be seen whether these gene products can be detected in biological fluids such as CSF as potential biomarkers in PD and LBD.

\section{Unbiased Methods in LBD Biomarker Discovery}

7.1. Genomics in PD and LBDs. As detailed above, traditional methods for molecular biomarker determination have been derived from targeted analyses of candidate genes/mutations 
and corresponding proteins in brain and body fluids such as CSF and blood, with the subsequent exploration of mechanisms in cell culture and animal models. An emerging alternate approach has been to evaluate genomes and proteomes with regard to specific neurodegenerative diseases and their components in an unbiased manner to yield a number of potential pathogenetic, therapeutic, and biomarker targets for further validation. With regard to the genomic analysis of the LBDs, gene expression profiling has proved to be a promising tool. Scherzer et al., for instance, examined transgenic Drosophila expressing the human $\alpha$-syn gene and performed temporal profiling of resultant gene expression [176]. They demonstrated a number of changes, including a downregulation of phospholipase A2 and other lipid genes, downregulation of several mitochondrial respiratory chain molecules, and alteration in membrane transport and energy genes such as voltage-gated calcium channel and lysosomal ATPase, suggesting that mitochondrial integrity might be affected by $\alpha$-syn overexpression.

In Parkinson's disease brain, RNA from populations of mesencephalic dopaminergic neurons with and without LB were isolated by immunolaser capture microdissection, amplified by polymerase chain reaction and expressed [177]. Interestingly, upregulation of the ubiquitin-specific protease 8 in LB-containing neurons indicated cellular damage and increased levels of ubiquitination in LB, whereas nonLB-bearing neurons showed increased expression of novel cytoprotective genes such as bullous pemphigoid antigen 1, an HSP-70-like gene (STCH) and Kelch-like 1. Although promising, further genomic profiling studies in DLB, PDD, and other $\mathrm{LBD}$ are needed to expand the range of novel gene targets for examination and validation.

7.2. Proteomic Profiling in PD and LBDs. As a complement to gene expression profiling and genomic methods, proteomic profiling has also assumed a greater importance in biomarker discovery for neurodegeneration with relevance to the LBD. Advances in methodologies such as 2-dimensional gel electrophoresis (2-D GE), liquid chromatography (LC), highresolution mass spectrometry (MS), and quantitative proteomics allow analysis of static or condition-dependent protein structure and function associated with PD and LBD in a variety of sample types such as brain or body fluids (reviewed in Shi et al. 2009) [178]. In mice treated with MPTP, a specific mitochondrial toxin, isotope-coded affinity tag assay of brain tissue followed by MS analysis revealed 100 proteins with significantly altered levels including many mitochondrial and metabolic molecules, $\beta$ APP and DJ-1 [179].

Basso et al. first examined the proteome of the substantia nigra from Parkinson's disease brain and age-matched controls [180]. Using 2D GE and peptide fingerprinting, of the 44 expressed proteins, 9 proteins differed in PD versus controls, including oxidative and mitochondrial proteins such as peroxiredoxin II, mitochondrial complex III, calcium channel, and others. A subsequent study in PD brain showed decreased frontal cortex levels of mortalin, a novel mitochondrial chaperone protein with roles in energy generation [181]. In addition, LBs isolated by laser-capture microdissection, were analyzed by LC/MS and ultimately demonstrated
156 candidate proteins involved in ubiquitin-proteasome system and synaptic function, from which the heat shock cognate-71, a chaperone involved in neurodegenerative disease, was identified and validated as a candidate target [182]. Abdi and colleagues carried out proteomic evaluation of CSF from $\mathrm{AD}, \mathrm{PD}$, and $\mathrm{DLB}$ patients and normal control individuals, using chromatography, MS, and isobaric tagging for relative and absolute quantification (iTRAQ), identifying numerous candidate proteins related to PD and DLB, such as lipoproteins ApoCl and ApoH [183]. Lastly, using surfaceenhanced laser desorption/ionization-time of flight (SELDITOF) MS analysis of serum from DLB patients compared to $\mathrm{AD}$, a combination of protein peaks provided the ability to separate DLB from non-DLB cases, with a sensitivity of $83.3 \%$ and a specificity of $95.8 \%$ [184]. Given promising findings, further exploration of the proteomics of the LBDs is warranted, and perhaps consideration should be given to determining whether combining various genomic and proteomic methods will be of value.

\section{Conclusions}

Over the last decade, tremendous advances have been made in understanding the pathogenetics of PD, PDD, and DLB, which has revealed not only the genetic basis of these disorders, but also related mechanisms common to all the LBD. In parallel, these discoveries have been a catalyst for translating and developing many of the involved proteins into promising biomarkers for disease. A common theme centers on genes that drive a complex network of synergistic and opposing cellular actions underlying pathogenesis. Aggregation of $\alpha$-syn, the main constituent of intracellular LBs, results in toxic oligomers and protofibrils which not only act intracellularly, but also are actively and passively released into the extracellular environment causing damage to surrounding tissue. Proinflammatory cytokines such as interleukins are also produced which perpetuates the inflammatory cascade. On the contrary, DJ-1, PINK1, parkin, and perhaps others molecules are upregulated to oppose cellular protein misfolding and oxidative stress and maintain mitochondrial function, while autophagy mechanisms attempt to limit the toxic effect of synucleins and other toxins by lysosomal engulfment and digestion. Much of this is reminiscent of a relatively new concept applied to infectious diseases and mechanical tissue injury termed "damage-associated molecular patterning" (DAMP), which is an evolved system to recognize, contain, and repair damage to cells and tissues. It is characterized by the abnormal release of molecules normally confined and operating within healthy cells or from foreign pathogenic agents, that when released into the extracellular space activate receptors and pathways leading to inflammation and multiplying cellular damage (reviewed by Bianchi) [185]. In this regard, events in the pathogenesis of PD, DLB, and related disorders may represent a novel variation of the DAMP response, and in a sense, biological fluid markers are therefore a measurement of DAMP activity as it relates to neurodegeneration.

Despite progress in developing biological markers for $\mathrm{PD}, \mathrm{PDD}$, and DLB, clinical diagnosis of this spectrum 
of disorders remains challenging. The need for highly sensitive and specific biomarkers that accurately mirror the underlying pathogenetic features of these disorders demands not only that more advanced detection methods be devised and validated in large sample populations, but also that novel biomarker candidates be selected for evaluation based on rational selection from the multiple-associated gene-mechanism associations in the LBD. Gene products including $\beta$ - and $\gamma$-synuclein, GBA, parkin, and PINK1 need to be examined in CSF, blood, and even urine to confirm their presence in biological fluids and threshold of detection. Alterations in the levels of these putative biomarker candidates in CSF and blood can provide further insight into the role these mechanisms may play in disease and also the ability of the potential biomarker to reflect resulting CNS changes. To better understand the relationship of gene mutations, mechanisms, and disease biomarkers in LBD, it would be of great interest to determine whether the levels of these putative biomarkers in CSF and peripheral fluids are altered in patients with known PD, DLB, and LBD mutations. It is likely that combinations of multiple peripheral biomarkers could be needed to monitor the various mechanistic aspects underlying the LBD, but the optimal combination has yet to be determined. Furthermore, both existing imaging modalities as well as novel imaging techniques to detect specific molecular biomarker targets will greatly complement peripheral biomarkers. New specific therapies for the LBD yet to be developed will probably target one or more of the multiple pathways described above, and indeed, this could determine which biomarker or combination of biomarkers would be appropriate as a therapeutic endpoint. Studies are also needed to establish which biomarkers will fulfill the criteria of minimum sensitivity and specificity for the LBD for consistent and reproducible diagnostic use in presymptomatic disease detection and also serve as robust tracking tools and endpoints in monitoring the efficacy of future LBD therapies.

\section{Acknowledgments}

This work was supported in part by a Grant-in-Aid for Science Research from the Ministry of Education, Culture, Sports, Science and Technology, Japan (to M. Hashimoto), by the Novartis Foundation for Gerontological Research (to M. Hashimoto), and by the following NIH Grants (to E. Masliah): AG18440, AG5131, AG022074, NS57096, and AG03197. The authors thank Dr. Michael Rafii, University of California, San Diego for his critical reading of their paper.

\section{References}

[1] I. G. McKeith, D. Galasko, K. Kosaka et al., "Consensus guidelines for the clinical and pathologic diagnosis of dementia with Lewy bodies (DLB): report of the consortium on DLB international workshop," Neurology, vol. 47, no. 5, pp. 1113-1124, 1996.

[2] A. R. Merdes, L. A. Hansen, D. V. Jeste et al., "Influence of Alzheimer pathology on clinical diagnostic accuracy in dementia with Lewy bodies," Neurology, vol. 60, no. 10, pp. 1586-1590, 2003.

[3] D. Weintraub, C. L. Comella, and S. Horn, "Parkinson's disease-Part 3: neuropsychiatric symptoms," American Journal of Managed Care, vol. 14, no. 2, pp. S59-S69, 2008.

[4] C. F. Lippa, J. E. Duda, M. Grossman et al., "DLB and PDD boundary issues: diagnosis, treatment, molecular pathology, and biomarkers," Neurology, vol. 68, no. 11, pp. 812-819, 2007.

[5] C. F. Lippa, T. W. Smith, and E. Perry, "Dementia with Lewy bodies: choline acetyltransferase parallels nucleus basalis pathology," Journal of Neural Transmission, vol. 106, no. 56, pp. 525-535, 1999.

[6] W. R. Markesbery, G. A. Jicha, H. Liu, and F. A. Schmitt, "Lewy body pathology in normal elderly subjects," Journal of Neuropathology and Experimental Neurology, vol. 68, no. 7, pp. 816-822, 2009.

[7] G. A. Jicha, F. A. Schmitt, E. Abner et al., "Prodromal clinical manifestations of neuropathologically confirmed Lewy body disease," Neurobiology of Aging, vol. 31, no. 10, pp. 18051813, 2010.

[8] C. R. Jack Jr., D. S. Knopman, W. J. Jagust et al., "Hypothetical model of dynamic biomarkers of the Alzheimer's pathological cascade," The Lancet Neurology, vol. 9, no. 1, pp. 119-128, 2010.

[9] B. Vellas, S. Andrieu, C. Sampaio, and G. Wilcock, "Diseasemodifying trials in Alzheimer's disease: a European task force consensus," The Lancet Neurology, vol. 6, no. 1, pp. 56-62, 2007.

[10] C. Haass and D. J. Selkoe, "Soluble protein oligomers in neurodegeneration: lessons from the Alzheimer's amyloid $\beta$ peptide," Nature Reviews Molecular Cell Biology, vol. 8, no. 2, pp. 101-112, 2007.

[11] A. J. Harding and G. M. Halliday, "Cortical Lewy body pathology in the diagnosis of dementia," Acta Neuropathologica, vol. 102, no. 4, pp. 355-363, 2001.

[12] P. H. St George-Hyslop, "Molecular genetics of Alzheimer's disease," Biological Psychiatry, vol. 47, no. 3, pp. 183-199, 2000.

[13] D. Selkoe and R. Kopan, "Notch and Presenilin: regulated intramembrane proteolysis links development and degeneration," Annual Review of Neuroscience, vol. 26, pp. 565-597, 2003.

[14] H. Vanderstichele, E. Van Kerschaver, C. Hesse et al., "Standardization of measurement of $\beta$-amyloid((1-42)) in cerebrospinal fluid and plasma," Amyloid, vol. 7, no. 4, pp. 245-258, 2000.

[15] L. Parnetti, P. Tiraboschi, A. Lanari et al., "Cerebrospinal fluid biomarkers in Parkinson's disease with dementia and dementia with Lewy bodies," Biological Psychiatry, vol. 64, no. 10, pp. 850-855, 2008.

[16] P. E. Spies, D. Slats, J. M. C. Sjögren et al., "The cerebrospinal fluid amyloid $\beta 42 / 40$ ratio in the differentiation of alzheimer's disease from non-alzheimer's dementia," Current Alzheimer Research, vol. 7, no. 5, pp. 470-476, 2010.

[17] W. E. Klunk, H. Engler, A. Nordberg et al., "Imaging brain amyloid in Alzheimer's disease with pittsburgh compoundB," Annals of Neurology, vol. 55, no. 3, pp. 306-319, 2004.

[18] C. C. Rowe, S. Ng, U. Ackermann et al., "Imaging $\beta$-amyloid burden in aging and dementia," Neurology, vol. 68, no. 20, pp. 1718-1725, 2007.

[19] S. N. Gomperts, D. M. Rentz, E. Moran et al., "Imaging amyloid deposition in Lewy body diseases," Neurology, vol. 71, no. 12, pp. 903-910, 2008. 
[20] M. Goedert, B. Ghetti, and M. G. Spillantini, "Tau gene mutations in frontotemporal dementia and parkinsonism linked to chromosome 17 (FTDP-17). Their relevance for understanding the neurogenerative process," Annals of the New York Academy of Sciences, vol. 920, pp. 74-83, 2000.

[21] D. G. Munoz, D. W. Dickson, C. Bergeron, I. R. A. Mackenzie, A. Delacourte, and V. Zhukareva, "The neuropathology and biochemistry of frontotemporal dementia," Annals of Neurology, vol. 54, supplement 5, pp. S24-S28, 2003.

[22] G. Basurto-Islas, J. Luna-Muñoz, A. L. Guillozet-Bongaarts, L. I. Binder, R. Mena, and F. García-Sierra, "Accumulation of aspartic acid- and glutamic acid -cleaved tau in neurofibrillary tangles correlates with progression in Alzheimer disease," Journal of Neuropathology and Experimental Neurology, vol. 67, no. 5, pp. 470-483, 2008.

[23] W. H. Stoothoff and G. V. W. Johnson, "Tau phosphorylation: physiological and pathological consequences," Biochimica et Biophysica Acta, vol. 1739, no. 2-3, pp. 280-297, 2005.

[24] H. Arai, Y. I. Morikawa, M. Higuchi et al., "Cerebrospinal fluid tau levels in neurodegenerative diseases with distinct tau-related pathology," Biochemical and Biophysical Research Communications, vol. 236, no. 2, pp. 262-264, 1997.

[25] L. Parnetti, A. Lanari, S. Amici, V. Gallai, E. Vanmechelen, and F. Hulstaert, "CSF phosphorylated tau is a possible marker for discriminating Alzheimer's disease from dementia with Lewy bodies. Phospho-Tau International Study Group," Neurological Sciences, vol. 22, no. 1, pp. 77-78, 2001.

[26] S. Engelborghs, K. De Vreese, T. Van de Casteele et al., "Diagnostic performance of a CSF-biomarker panel in autopsy-confirmed dementia," Neurobiology of Aging, vol. 29, no. 8, pp. 1143-1159, 2008.

[27] K. Buerger, R. Zinkowski, S. J. Teipel et al., "Differential diagnosis of Alzheimer disease with cerebrospinal fluid levels of tau protein phosphorylated at threonine 231," Archives of Neurology, vol. 59, no. 8, pp. 1267-1272, 2002.

[28] D. de Jong, R. W. M. M. Jansen, Y. A. L. Pijnenburg et al., "CSF neurofilament proteins in the differential diagnosis of dementia," Journal of Neurology, Neurosurgery and Psychiatry, vol. 78, no. 9, pp. 936-938, 2007.

[29] H. Hampel, K. Buerger, R. Zinkowski et al., "Measurement of phosphorylated tau epitopes in the differential diagnosis of Alzheimer disease: a comparative cerebrospinal fluid study," Archives of General Psychiatry, vol. 61, no. 1, pp. 95-102, 2004.

[30] K. Koopman, N. Le Bastard, J. J. Martin, G. Nagels, P. P. De Deyn, and S. Engelborghs, "Improved discrimination of autopsy-confirmed Alzheimer's disease (AD) from nonAD dementias using CSF P-tau(181P)," Neurochemistry International, vol. 55, no. 4, pp. 214-218, 2009.

[31] J. M. George, H. Jin, W. S. Woods, and D. F. Clayton, "Characterization of a novel protein regulated during the critical period for song learning in the zebra finch," Neuron, vol. 15, no. 2, pp. 361-372, 1995.

[32] M. A. Alim, M. S. Hossain, K. Arima et al., "Tubulin seeds $\alpha$-synuclein fibril formation," Journal of Biological Chemistry, vol. 277, no. 3, pp. 2112-2117, 2002.

[33] M. A. Alim, Q. L. Ma, K. Takeda et al., "Demonstration of a role for $\alpha$-synuclein as a functional microtubule-associated protein," Journal of Alzheimer's Disease, vol. 6, no. 4, pp. 435442,2004

[34] N. M. Bonini and B. I. Giasson, "Snaring the function of $\alpha$ synuclein,” Cell, vol. 123, no. 3, pp. 359-361, 2005.

[35] A. A. Cooper, A. D. Gitler, A. Cashikar et al., " $\alpha$-synuclein blocks ER-Golgi traffic and Rabl rescues neuron loss in
Parkinson's models," Science, vol. 313, no. 5785, pp. 324-328, 2006.

[36] G. Liu, C. Zhang, J. Yin et al., “ $\alpha$-synuclein is differentially expressed in mitochondria from different rat brain regions and dose-dependently down-regulates complex I activity," Neuroscience Letters, vol. 454, no. 3, pp. 187-192, 2009.

[37] J. Madine, A. J. Doig, and D. A. Middleton, "A study of the regional effects of $\alpha$-synuclein on the organization and stability of phospholipid bilayers," Biochemistry, vol. 45, no. 18, pp. 5783-5792, 2006.

[38] M. Zhu, Z. J. Qin, D. Hu, L. A. Munishkina, and A. L. Fink, " $\alpha$-synuclein can function as an antioxidant preventing oxidation of unsaturated lipid in vesicles," Biochemistry, vol. 45, no. 26, pp. 8135-8142, 2006.

[39] O. M. A. El-Agnaf and G. B. Irvine, "Aggregation and neurotoxicity of $\alpha$-synuclein and related peptides," Biochemical Society Transactions, vol. 30, no. 4, pp. 559-565, 2002.

[40] H. I. Hurtig, J. Q. Trojanowski, J. Galvin et al., "Alphasynuclein cortical Lewy bodies correlate with dementia in Parkinson's disease," Neurology, vol. 54, no. 10, pp. 19161921, 2000.

[41] M. G. Spillantini, R. A. Crowther, R. Jakes, M. Hasegawa, and M. Goedert, “ $\alpha$-synuclein in filamentous inclusions of Lewy bodies from Parkinson's disease and dementia with Lewy bodies," Proceedings of the National Academy of Sciences of the United States of America, vol. 95, no. 11, pp. 6469-6473, 1998.

[42] M. Hashimoto, L. J. Hsu, A. Sisk et al., "Human recombinant $\mathrm{NACP} / \alpha$-synuclein is aggregated and fibrillated in vitro: relevance for Lewy body disease," Brain Research, vol. 799, no. 2, pp. 301-306, 1998.

[43] M. H. Polymeropoulos, C. Lavedan, E. Leroy et al., "Mutation in the $\alpha$-synuclein gene identified in families with Parkinson's disease," Science, vol. 276, no. 5321, pp. 2045-2047, 1997.

[44] R. Krüger, W. Kuhn, T. Müller et al., "Ala30Pro mutation in the gene encoding $\alpha$-synuclein in Parkinson's disease," Nature Genetics, vol. 18, no. 2, pp. 106-108, 1998.

[45] L. I. Golbe, G. Di Iorio, G. Sanges et al., "Clinical genetic analysis of Parkinson's disease in the Contursi kindred," Annals of Neurology, vol. 40, no. 5, pp. 767-775, 1996.

[46] K. Yamaguchi, E. J. Cochran, J. R. Murrell et al., "Abundant neuritic inclusions and microvacuolar changes in a case of diffuse Lewy body disease with the A53T mutation in the $\alpha$ synuclein gene," Acta Neuropathologica, vol. 110, no. 3, pp. 298-305, 2005

[47] L. Morfis and D. J. Cordato, "Dementia with Lewy bodies in an elderly Greek male due to $\alpha$-synuclein gene mutation," Journal of Clinical Neuroscience, vol. 13, no. 9, pp. 942-944, 2006.

[48] J. J. Zarranz, J. Alegre, J. C. Gómez-Esteban et al., “The new mutation, E46K, of $\alpha$-synuclein causes Parkinson and Lewy body dementia," Annals of Neurology, vol. 55, no. 2, pp. 164173, 2004.

[49] A. B. Singleton, M. Farrer, J. Johnson et al., " $\alpha$-synuclein locus triplication causes Parkinson's disease," Science, vol. 302, no. 5646, p. 841, 2003.

[50] J. Li, V. N. Uversky, and A. L. Fink, "Effect of familial Parkinson's disease point mutations A30P and A53T on the structural properties, aggregation, and fibrillation of human $\alpha$-synuclein," Biochemistry, vol. 40, no. 38, pp. 11604-11613, 2001.

[51] E. Tsika, M. Moysidou, J. Guo et al., "Distinct region-specific $\alpha$-synuclein oligomers in A53T transgenic mice: implications for neurodegeneration," Journal of Neuroscience, vol. 30, no. 9, pp. 3409-3418, 2010. 
[52] R. A. Fredenburg, C. Rospigliosi, R. K. Meray et al., "The impact of the E46K mutation on the properties of $\alpha$ synuclein in its monomelic and oligomeric states," Biochemistry, vol. 46, no. 24, pp. 7107-7118, 2007.

[53] A. Iwai, E. Masliah, M. Yoshimoto et al., "The precursor protein of non- $\mathrm{A} \beta$ component of Alzheimer's disease amyloid is a presynaptic protein of the central nervous system," Neuron, vol. 14, no. 2, pp. 467-475, 1995.

[54] S. Nakajo, S. Shioda, Y. Nakai, and K. Nakaya, "Localization of phosphoneuroprotein 14 (PNP 14) and its mRNA expression in rat brain determined by immunocytochemistry and in situ hybridization," Molecular Brain Research, vol. 27, no. 1, pp. 81-86, 1994.

[55] M. Hashimoto, E. Rockenstein, M. Mante, M. Mallory, and E. Masliah, " $\beta$-synuclein inhibits $\alpha$-synuclein aggregation: a possible role as an anti-Parkinsonian factor," Neuron, vol. 32, no. 2, pp. 213-223, 2001.

[56] H. Ohtake, P. Limprasert, Y. Fan et al., " $\beta$-synuclein gene alterations in dementia with Lewy bodies," Neurology, vol. 63, no. 5, pp. 805-811, 2004.

[57] M. Fujita, S. Sugama, K. Sekiyama et al., "A $\beta$-synuclein mutation linked to dementia produces neurodegeneration when expressed in mouse brain," Nature Communications, vol. 1, no. 8, article 110, 2010.

[58] J. Wei, M. Fujita, M. Nakai et al., "Enhanced lysosomal pathology caused by $\beta$-synuclein mutants linked to dementia with Lewy bodies," Journal of Biological Chemistry, vol. 282, no. 39, pp. 28904-28914, 2007.

[59] V. L. Buchman, H. J. Hunter, L. G. Pinon et al., "Persyn, a member of the synuclein family, has a distinct pattern of expression in the developing nervous system," Journal of Neuroscience, vol. 18, no. 22, pp. 9335-9341, 1998.

[60] M. Ahmad, S. Attoub, M. N. Singh, F. L. Martin, and O. M. A. El-Agnaf, " $\gamma$-synuclein and the progression of cancer," Journal of the Federation of American Societies for Experimental Biology, vol. 21, no. 13, pp. 3419-3430, 2007.

[61] K. Nishioka, C. Wider, C. Vilariño-Güell et al., "Association of $\alpha-, \beta$, and $\gamma$-synuclein with diffuse Lewy body disease," Archives of Neurology, vol. 67, no. 8, pp. 970-975, 2010.

[62] J. E. Galvin, K. Uryu, V. M. Y. Lee, and J. Q. Trojanowski, "Axon pathology in Parkinson's disease and Lewy body dementia hippocampus contains $\alpha$-, $\beta$-, and $\gamma$-synuclein," Proceedings of the National Academy of Sciences of the United States of America, vol. 96, no. 23, pp. 13450-13455, 1999.

[63] N. Ninkina, O. Peters, S. Millership, H. Salem, H. van der Putten, and V. L. Buchman, " $\gamma$-synucleinopathy: neurodegeneration associated with overexpression of the mouse protein," Human Molecular Genetics, vol. 18, no. 10, pp. 1779-1794, 2009.

[64] V. L. Buchman, J. Adu, L. G. P. Pinõn, N. N. Ninkina, and A. M. Davies, "Persyn, a member of the synuclein family, influences neurofilament network integrity," Nature Neuroscience, vol. 1, no. 2, pp. 101-103, 1998.

[65] O. M. El-Agnaf, S. A. Salem, K. E. Paleologou et al., "Alphasynuclein implicated in Parkinson's disease is present in extracellular biological fluids, including human plasma," Journal of the Federation of American Societies for Experimental Biology, vol. 17, no. 13, pp. 1945-1947, 2003.

[66] H. J. Lee, S. Patel, and S. J. Lee, "Intravesicular localization and exocytosis of $\alpha$-synuclein and its aggregates," Journal of Neuroscience, vol. 25, no. 25, pp. 6016-6024, 2005.

[67] P. Desplats, H. J. Lee, E. J. Bae et al., "Inclusion formation and neuronal cell death through neuron-to-neuron transmission of $\alpha$-synuclein," Proceedings of the National Academy of Sciences of the United States of America, vol. 106, no. 31, pp. 13010-13015, 2009.

[68] J. Y. Sung, S. M. Park, C. H. Lee et al., "Proteolytic cleavage of extracellular secreted $\alpha$-synuclein via matrix metalloproteinases," Journal of Biological Chemistry, vol. 280, no. 26, pp. 25216-25224, 2005.

[69] R. Borghi, R. Marchese, A. Negro et al., "Full length $\alpha$ synuclein is present in cerebrospinal fluid from Parkinson's disease and normal subjects," Neuroscience Letters, vol. 287, no. 1, pp. 65-67, 2000.

[70] B. Mollenhauer, V. Cullen, I. Kahn et al., "Direct quantification of CSF $\alpha$-synuclein by ELISA and first cross-sectional study in patients with neurodegeneration," Experimental Neurology, vol. 213, no. 2, pp. 315-325, 2008.

[71] E. B. Mukaetova-Ladinska, J. Milne, A. Andras et al., "Alphaand gamma-synuclein proteins are present in cerebrospinal fluid and are increased in aged subjects with neurodegenerative and vascular changes," Dementia and Geriatric Cognitive Disorders, vol. 26, no. 1, pp. 32-42, 2008.

[72] Z. Hong, M. Shi, K. A. Chung et al., "DJ-1 and $\alpha$-synuclein in human cerebrospinal fluid as biomarkers of Parkinson's disease," Brain, vol. 133, no. 3, pp. 713-726, 2010.

[73] C. Ballard, E. L. Jones, E. Londos, L. Minthon, P. Francis, and D. Aarsland, " $\alpha$-synuclein antibodies recognize a protein present at lower levels in the CSF of patients with dementia with Lewy bodies," International Psychogeriatrics, vol. 22, no. 2, pp. 321-327, 2010.

[74] T. Tokuda, M. M. Qureshi, M. T. Ardah et al., "Detection of elevated levels of $\alpha$-synuclein oligomers in CSF from patients with Parkinson disease," Neurology, vol. 75, no. 20, pp. 17661772, 2010.

[75] Q. X. Li, S. S. Mok, K. M. Laughton et al., "Plasma $\alpha$ synuclein is decreased in subjects with Parkinson's disease," Experimental Neurology, vol. 204, no. 2, pp. 583-588, 2007.

[76] O. M. A. El-Agnaf, S. A. Salem, K. E. Paleologou et al., "Detection of oligomeric forms of $\alpha$-synuclein protein in human plasma as a potential biomarker for Parkinson's disease," Journal of the Federation of American Societies for Experimental Biology, vol. 20, no. 3, pp. 419-425, 2006.

[77] A. W. Michell, L. M. Luheshi, and R. A. Barker, "Skin and platelet $\alpha$-synuclein as peripheral biomarkers of Parkinson's disease," Neuroscience Letters, vol. 381, no. 3, pp. 294-298, 2005.

[78] R. Barbour, K. Kling, J. P. Anderson et al., "Red blood cells are the major source of alpha-synuclein in blood," Neurodegenerative Diseases, vol. 5, no. 2, pp. 55-59, 2008.

[79] D. Nagakubo, T. Taira, H. Kitaura et al., "DJ-1, a novel oncogene which transforms mouse NIH3T3 cells in cooperation with ras," Biochemical and Biophysical Research Communications, vol. 231, no. 2, pp. 509-513, 1997.

[80] S. Shendelman, A. Jonason, C. Martinat, T. Leete, and A. Abeliovich, "DJ-1 Is a redox-dependent molecular chaperone that inhibits $\alpha$-synuclein aggregate formation," Public Library of Science, Biology, vol. 2, no. 11, article e362, 2004.

[81] K. Takahashi, T. Taira, T. Niki, C. Seino, S. M. M. IguchiAriga, and H. Ariga, "DJ-1 positively regulates the androgen receptor by impairing the binding of PIASx $\alpha$ to the receptor," Journal of Biological Chemistry, vol. 276, no. 40, pp. 3755637563, 2001.

[82] Y. Shinbo, T. Niki, T. Taira et al., "Proper SUMO-1 conjugation is essential to DJ-1 to exert its full activities," Cell Death and Differentiation, vol. 13, no. 1, pp. 96-108, 2006. 
[83] R. H. Kim, P. D. Smith, H. Aleyasin et al., "Hypersensitivity of DJ-1-deficient mice to 1-methyl-4-phenyl-1,2,3,6-tetrahydropyrindine (MPTP) and oxidative stress," Proceedings of the National Academy of Sciences of the United States of America, vol. 102, no. 14, pp. 5215-5220, 2005.

[84] M. Meulener, A. J. Whitworth, C. E. Armstrong-Gold et al., "Drosophila DJ-1 mutants are selectively sensitive to environmental toxins associated with Parkinson's disease," Current Biology, vol. 15, no. 17, pp. 1572-1577, 2005.

[85] V. Bonifati, P. Rizzu, M. J. van Baren et al., "Mutations in the DJ-1 gene associated with autosomal recessive early-onset parkinsonism," Science, vol. 299, no. 5604, pp. 256-259, 2003.

[86] D. J. Moore, L. Zhang, T. M. Dawson, and V. L. Dawson, "A missense mutation (L166P) in DJ-1, linked to familial Parkinson's disease, confers reduced protein stability and impairs homo-oligomerization," Journal of Neurochemistry, vol. 87, no. 6, pp. 1558-1567, 2003.

[87] D. W. Miller, R. Ahmad, S. Hague et al., "L166P mutant DJ-1, causative for recessive Parkinson's disease, is degraded through the ubiquitin-proteasome system," Journal of Biological Chemistry, vol. 278, no. 38, pp. 36588-36595, 2003.

[88] A. Arias Vásquez, K. Sleegers, M. C. J. Dekker et al., "A deletion in DJ-1 and the risk of dementia-a populationbased survey," Neuroscience Letters, vol. 372, no. 3, pp. 196199, 2004.

[89] C. M. Morris, K. K. O’Brien, A. M. Gibson, J. A. Hardy, and A. B. Singleton, "Polymorphism in the human DJ-1 gene is not associated with sporadic dementia with Lewy bodies or Parkinson's disease," Neuroscience Letters, vol. 352, no. 2, pp. 151-153, 2003.

[90] P. Rizzu, D. A. Hinkle, V. Zhukareva et al., "DJ-1 colocalizes with tau inclusions: a link between parkinsonism and dementia," Annals of Neurology, vol. 55, no. 1, pp. 113-118, 2004.

[91] Y. Wang, J. S. Chandran, H. Cai, and M. P. Mattson, "DJ1 is essential for long-term depression at hippocampal CA1 synapses," NeuroMolecular Medicine, vol. 10, no. 1, pp. 4045, 2008.

[92] M. Waragai, J. Wei, M. Fujita et al., "Increased level of DJ-1 in the cerebrospinal fluids of sporadic Parkinson's disease," Biochemical and Biophysical Research Communications, vol. 345, no. 3, pp. 967-972, 2006.

[93] M. Waragai, M. Nakai, J. Wei et al., "Plasma levels of DJ-1 as a possible marker for progression of sporadic Parkinson's disease," Neuroscience Letters, vol. 425, no. 1, pp. 18-22, 2007.

[94] L. Allard, P. R. Burkhard, P. Lescuyer et al., "PARK7 and nucleoside diphosphate kinase A as plasma markers for the early diagnosis of stroke," Clinical Chemistry, vol. 51, no. 11, pp. 2043-2051, 2005.

[95] S. Koide-Yoshida, T. Niki, M. Ueda et al., "DJ-1 degrades transthyretin and an inactive form of DJ-1 is secreted in familial amyloidotic polyneuropathy," International Journal of Molecular Medicine, vol. 19, no. 6, pp. 885-893, 2007.

[96] H. Liu, M. Wang, M. Li et al., "Expression and role of DJ-1 in leukemia," Biochemical and Biophysical Research Communications, vol. 375, no. 3, pp. 477-483, 2008.

[97] J. Choi, M. C. Sullards, J. A. Olzmann et al., "Oxidative damage of DJ-1 is linked to sporadic Parkinson and Alzheimer diseases," Journal of Biological Chemistry, vol. 281, no. 16, pp. 10816-10824, 2006.

[98] E. Sidransky, "Gaucher disease: complexity in a "simple" disorder," Molecular Genetics and Metabolism, vol. 83, no. 12, pp. 6-15, 2004.
[99] O. Goker-Alpan, R. Schiffmann, M. E. LaMarca, R. L. Nussbaum, A. McInerney-Leo, and E. Sidransky, "Parkinsonism among Gaucher disease carriers," Journal of Medical Genetics, vol. 41, no. 12, pp. 937-940, 2004.

[100] A. Halperin, D. Elstein, and A. Zimran, "Increased incidence of Parkinson disease among relatives of patients with Gaucher disease," Blood Cells, Molecules, and Diseases, vol. 36, no. 3, pp. 426-428, 2006.

[101] J. Aharon-Peretz, S. Badarny, H. Rosenbaum, and R. Gershoni-Baruch, "Mutations in the glucocerebrosidase gene and Parkinson disease: phenotype-genotype correlation," Neurology, vol. 65, no. 9, pp. 1460-1461, 2005.

[102] J. Aharon-Peretz, H. Rosenbaum, and R. Gershoni-Baruch, "Mutations in the glucocerebrosidase gene and Parkinson's disease in Ashkenazi Jews," The New England Journal of Medicine, vol. 351, no. 19, pp. 1972-1977, 2004.

[103] A. Lwin, E. Orvisky, O. Goker-Alpan, M. E. LaMarca, and E. Sidransky, "Glucocerebrosidase mutations in subjects with parkinsonism," Molecular Genetics and Metabolism, vol. 81, no. 1, pp. 70-73, 2004.

[104] Z. Gan-Or, N. Giladi, U. Rozovski et al., "Genotypephenotype correlations between GBA mutations and Parkinson disease risk and onset," Neurology, vol. 70, no. 24, pp. 2277-2283, 2008.

[105] E. Sidransky, M. A. Nalls, J. O. Aasly et al., "Multicenter analysis of glucocerebrosidase mutations in Parkinson's disease," The New England Journal of Medicine, vol. 361, no. 17, pp. 1651-1661, 2009.

[106] J. Neumann, J. Bras, E. Deas et al., "Glucocerebrosidase mutations in clinical and pathologically proven Parkinson's disease," Brain, vol. 132, no. 7, pp. 1783-1794, 2009.

[107] Z. Gan-Or, N. Giladi, and A. Orr-Urtreger, "Differential phenotype in Parkinson's disease patients with severe versus mild GBA mutations," Brain, vol. 132, no. 10, article e125, 2009.

[108] L. N. Clark, L. A. Kartsaklis, R. W. Gilbert et al., "Association of glucocerebrosidase mutations with dementia with Lewy bodies," Archives of Neurology, vol. 66, no. 5, pp. 578-583, 2009.

[109] O. Goker-Alpan, B. I. Giasson, M. J. Eblan et al., "Glucocerebrosidase mutations are an important risk factor for Lewy body disorders," Neurology, vol. 67, no. 5, pp. 908-910, 2006.

[110] L. Alvarez-Erviti, M. C. Rodriguez-Oroz, J. M. Cooper et al., "Chaperone-mediated autophagy markers in Parkinson disease brains," Archives of Neurology, vol. 67, no. 12, pp. 1464-1472, 2010.

[111] A. M. Cuervo, L. Stafanis, R. Fredenburg, P. T. Lansbury, and D. Sulzer, "Impaired degradation of mutant $\alpha$-synuclein by chaperone-mediated autophagy," Science, vol. 305, no. 5688, pp. 1292-1295, 2004.

[112] S. K. Mak, A. L. McCormack, A. B. Manning-Bog, A. M. Cuervo, and D. A. Di Monte, "Lysosomal degradation of $\alpha$ synuclein in vivo," Journal of Biological Chemistry, vol. 285, no. 18, pp. 13621-13629, 2010.

[113] K. Hruska, O. Goker-Alpan, and E. Sidransky, "Gaucher disease and the synucleinopathies," Journal of Biomedicine and Biotechnology, vol. 2006, no. 3, Article ID 78549, 2006.

[114] K. Wong, E. Sidransky, A. Verma et al., "Neuropathology provides clues to the pathophysiology of Gaucher disease," Molecular Genetics and Metabolism, vol. 82, no. 3, pp. 192207, 2004.

[115] I. Ron, D. Rapaport, and M. Horowitz, "Interaction between parkin and mutant glucocerebrosidase variants: a possible link between Parkinson disease and Gaucher disease," 
Human Molecular Genetics, vol. 19, no. 19, pp. 3771-3781, 2010.

[116] M. Migita, H. Hamada, J. Fujimura, A. Watanabe, T. Shimada, and Y. Fukunaga, "Glucocerebrosidase level in the cerebrospinal fluid during enzyme replacement therapyunsuccessful treatment of the neurological abnormality in type 2 Gaucher disease," European Journal of Pediatrics, vol. 162, no. 7-8, pp. 524-525, 2003.

[117] C. Balducci, L. Pierguidi, E. Persichetti et al., "Lysosomal hydrolases in cerebrospinal fluid from subjects with Parkinson's disease," Movement Disorders, vol. 22, no. 10, pp. 14811484, 2007.

[118] L. Parnetti, C. Balducci, L. Pierguidi et al., "Cerebrospinal fluid $\beta$-glucocerebrosidase activity is reduced in dementia with Lewy Bodies," Neurobiology of Disease, vol. 34, no. 3, pp. 484-486, 2009.

[119] G. J. Ho, R. Drego, E. Hakimian, and E. Masliah, "Mechanisms of cell signaling and inflammation in Alzheimer's disease," Current Drug Targets_Inflammation and Allergy, vol. 4, no. 2, pp. 247-256, 2005.

[120] K. M. Mattila, J. O. Rinne, T. Lehtimäki, M. Röyttä, J. P. Ahonen, and M. Hurme, "Association of an interleukin 1B gene polymorphism (-511) with Parkinson's disease in Finnish patients," Journal of Medical Genetics, vol. 39, no. 6, pp. 400-402, 2002.

[121] A. D. Wahner, J. S. Sinsheimer, J. M. Bronstein, and B. Ritz, "Inflammatory cytokine gene polymorphisms and increased risk of Parkinson disease," Archives of Neurology, vol. 64, no. 6, pp. 836-840, 2007.

[122] M. Nishimura, I. Mizuta, E. Mizuta, S. Yamasaki, M. Ohta, and S. Kuno, "Influence of interleukin- $1 \beta$ gene polymorphisms on age-at-onset of sporadic Parkinson's disease," Neuroscience Letters, vol. 284, no. 1-2, pp. 73-76, 2000.

[123] X. Su, K. A. Maguire-Zeiss, R. Giuliano, L. Prifti, K. Venkatesh, and H. J. Federoff, "Synuclein activates microglia in a model of Parkinson's disease," Neurobiology of Aging, vol. 29, no. 11, pp. 1690-1701, 2008.

[124] D. Blum-Degena, T. Müller, W. Kuhn, M. Gerlach, H. Przuntek, and P. Riederer, "Interleukin- $1 \beta$ and interleukin-6 are elevated in the cerebrospinal fluid of Alzheimer's and de novo Parkinson's disease patients," Neuroscience Letters, vol. 202, no. 1-2, pp. 17-20, 1995.

[125] T. Müller, D. Blum-Degen, H. Przuntek, and W. Kuhn, "Interleukin-6 levels in cerebrospinal fluid inversely correlate to severity of Parkinson's disease," Acta Neurologica Scandinavica, vol. 98, no. 2, pp. 142-144, 1998.

[126] M. Mogi, M. Harada, P. Riederer, H. Narabayashi, K. Fujita, and T. Nagatsu, "Tumor necrosis factor- $\alpha$ (TNF- $\alpha$ ) increases both in the brain and in the cerebrospinal fluid from parkinsonian patients," Neuroscience Letters, vol. 165, no. 12, pp. 208-210, 1994.

[127] H. Chen, E. J. O’Reilly, M. A. Schwarzschild, and A. Ascherio, "Peripheral inflammatory biomarkers and risk of Parkinson's disease," American Journal of Epidemiology, vol. 167, no. 1, pp. 90-95, 2008.

[128] E. Gómez-Tortosa, I. Gonzalo, S. Fanjul et al., “Cerebrospinal fluid markers in dementia with Lewy bodies compared with Alzheimer disease," Archives of Neurology, vol. 60, no. 9, pp. 1218-1222, 2003.

[129] Q. Liu, F. Xie, S. L. Siedlak et al., "Neurofilament proteins in neurodegenerative diseases," Cellular and Molecular Life Sciences, vol. 61, no. 24, pp. 3057-3075, 2004.
[130] C. Lavedan, S. Buchholtz, R. L. Nussbaum, R. L. Albin, and M. H. Polymeropoulos, "A mutation in the human neurofilament M gene in Parkinson's disease that suggests a role for the cytoskeleton in neuronal degeneration," Neuroscience Letters, vol. 322, no. 1, pp. 57-61, 2002.

[131] M. L. Schmidt, J. Murray, V. M. Y. Lee, W. D. Hill, A. Wertkin, and J. Q. Trojanowski, "Epitope map of neurofilament protein domains in cortical and peripheral nervous system Lewy bodies," American Journal of Pathology, vol. 139, no. 1, pp. 53-65, 1991.

[132] B. Holmberg, L. Rosengren, J. E. Karlsson, and B. Johnels, "Increased cerebrospinal fluid levels of neurofilament protein in progressive supranuclear palsy and multiple-system atrophy compared with Parkinson's disease," Movement Disorders, vol. 13, no. 1, pp. 70-77, 1998.

[133] H. Tohgi, T. Abe, K. Hashiguchi, M. Saheki, and S. Takahashi, "Remarkable reduction in acetylcholine concentration in the cerebrospinal fluid from patients with Alzheimer type dementia," Neuroscience Letters, vol. 177, no. 1-2, pp. 139$142,1994$.

[134] M. J. Welch, C. H. Markham, and D. J. Jenden, "Acetylcholine and choline in cerebrospinal fluid of patients with Parkinson's disease and Huntington's chorea," Journal of Neurology Neurosurgery and Psychiatry, vol. 39, no. 4, pp. 367-374, 1976.

[135] H. Shimada, S. Hirano, H. Shinotoh et al., "Mapping of brain acetylcholinesterase alterations in Lewy body disease by PET," Neurology, vol. 73, no. 4, pp. 273-278, 2009.

[136] G. Lunardi, S. Galati, D. Tropepi et al., "Correlation between changes in CSF dopamine turnover and development of dyskinesia in Parkinson's disease," Parkinsonism and Related Disorders, vol. 15, no. 5, pp. 383-389, 2009.

[137] K. Kanemaru and H. Yamanouchi, "Assessment of CSF homovanillic acid levels distinguishes dementia with Lewy bodies from Alzheimer's disease," Journal of Neurology, vol. 249, no. 8, pp. 1125-1126, 2002.

[138] X. S. Hu, N. Okamura, H. Arai et al., "18F-fluorodopa PET study of striatal dopamine uptake in the diagnosis of dementia with Lewy bodies," Neurology, vol. 55, no. 10, pp. 1575-1577, 2000.

[139] Z. Walker, E. Jaros, R. W. H. Walker et al., "Dementia with Lewy bodies: a comparison of clinical diagnosis, FP-CIT single photon emission computed tomography imaging and autopsy," Journal of Neurology, Neurosurgery and Psychiatry, vol. 78, no. 11, pp. 1176-1181, 2007.

[140] J. T. O’Brien, S. Colloby, J. Fenwick et al., "Dopamine transporter loss visualized with FP-CIT SPECT in the differential diagnosis of dementia with Lewy bodies," Archives of Neurology, vol. 61, no. 6, pp. 919-925, 2004.

[141] I. McKeith, J. O'Brien, Z. Walker et al., "Sensitivity and specificity of dopamine transporter imaging with I-FPCIT SPECT in dementia with Lewy bodies: a phase III, multicentre study," The Lancet Neurology, vol. 6, no. 4, pp. 305-313, 2007.

[142] F. Roselli, N. M. Pisciotta, R. Perneczky et al., "Severity of neuropsychiatric symptoms and dopamine transporter levels in dementia with Lewy bodies: a 123I-FP-CIT SPECT study," Movement Disorders, vol. 24, no. 14, pp. 2097-2103, 2009.

[143] M. Yoshita, J. Taki, and M. Yamada, "A clinical role for [(123)I]MIBG myocardial scintigraphy in the distinction between dementia of the Alzheimer's-type and dementia with Lewy bodies," Journal of Neurology Neurosurgery and Psychiatry, vol. 71, no. 5, pp. 583-588, 2001. 
[144] K. Wada-Isoe, M. Kitayama, K. Nakaso, and K. Nakashima, "Diagnostic markers for diagnosing dementia with Lewy bodies: CSF and MIBG cardiac scintigraphy study," Journal of the Neurological Sciences, vol. 260, no. 1-2, pp. 33-37, 2007.

[145] H. Watanabe, T. Ieda, T. Katayama et al., "Cardiac (123)Imeta-iodobenzylguanidine (MIBG) uptake in dementia with Lewy bodies: comparison with Alzheimer's disease," Journal of Neurology Neurosurgery and Psychiatry, vol. 70, no. 6, pp. 781-783, 2001.

[146] M. Yoshita, J. Taki, K. Yokoyama et al., "Value of 123I-MIBG radioactivity in the differential diagnosis of DLB from AD," Neurology, vol. 66, no. 12, pp. 1850-1854, 2006.

[147] H. Hanyu, S. Shimizu, K. Hirao et al., "Comparative value of brain perfusion SPECT and [(123)I]MIBG myocardial scintigraphy in distinguishing between dementia with Lewy bodies and Alzheimer's disease," European Journal of Nuclear Medicine and Molecular Imaging, vol. 33, no. 3, pp. 248-253, 2006.

[148] M. Estorch, V. Camacho, P. Paredes et al., "Cardiac (123)Imetaiodobenzylguanidine imaging allows early identification of dementia with Lewy bodies during life," European Journal of Nuclear Medicine and Molecular Imaging, vol. 35, no. 9, pp. 1636-1641, 2008.

[149] S. Kobayashi, M. Tateno, H. Morii, K. Utsumi, and T. Saito, "Decreased cardiac MIBG uptake, its correlation with clinical symptoms in dementia with Lewy bodies," Psychiatry Research, vol. 174, no. 1, pp. 76-80, 2009.

[150] R. Watson, A. M. Blamire, and J. T. O'Brien, "Magnetic resonance imaging in Lewy body dementias," Dementia and Geriatric Cognitive Disorders, vol. 28, no. 6, pp. 493-506, 2009.

[151] E. J. Burton, I. G. McKeith, D. J. Burn, E. D. Williams, and J. T. O'Brien, "Cerebral atrophy in Parkinson's disease with and without dementia: a comparison with Alzheimer's disease, dementia with Lewy bodies and controls," Brain, vol. 127, no. 4, pp. 791-800, 2004.

[152] J. T. O’Brien, S. Paling, R. Barber et al., "Progressive brain atrophy on serial MRI in dementia with Lewy bodies, AD, and vascular dementia," Neurology, vol. 56, no. 10, pp. 13861388, 2001.

[153] E. J. Burton, I. G. McKeith, D. J. Burn, and J. T. O’Brien, "Brain atrophy rates in Parkinson's disease with and without dementia using serial magnetic resonance imaging," Movement Disorders, vol. 20, no. 12, pp. 1571-1576, 2005.

[154] F. Sabattoli, M. Boccardi, S. Galluzzi, A. Treves, P. M. Thompson, and G. B. Frisoni, "Hippocampal shape differences in dementia with Lewy bodies," NeuroImage, vol. 41, no. 3, pp. 699-705, 2008.

[155] M. J. Firbank, A. M. Blamire, M. S. Krishnan et al., "Diffusion tensor imaging in dementia with Lewy bodies and Alzheimer's disease," Psychiatry Research, vol. 155, no. 2, pp. 135-145, 2007.

[156] K. Lobotesis, J. D. Fenwick, A. Phipps et al., "Occipital hypoperfusion on SPECT in dementia with Lewy bodies but not AD," Neurology, vol. 56, no. 5, pp. 643-649, 2001.

[157] S. J. Colloby, J. D. Fenwick, D. E. Williams et al., "A comparison of (99m)Tc-HMPAO SPET changes in dementia with Lewy bodies and Alzheimer's disease using statistical parametric mapping," European Journal of Nuclear Medicine and Molecular Imaging, vol. 29, no. 5, pp. 615-622, 2002.

[158] J. Hardy, P. Lewis, T. Revesz, A. Lees, and C. Paisan-Ruiz, "The genetics of Parkinson's syndromes: a critical review," Current Opinion in Genetics and Development, vol. 19, no. 3, pp. 254-265, 2009.
[159] D. MacLeod, J. Dowman, R. Hammond, T. Leete, K. Inoue, and A. Abeliovich, "The familial parkinsonism gene LRRK2 regulates neurite process morphology," Neuron, vol. 52, no. 4, pp. 587-593, 2006.

[160] C. H. Lin, P. I. Tsai, R. M. Wu, and C. T. Chien, "LRRK2 G2019S mutation induces dendrite degeneration through mislocalization and phosphorylation of tau by recruiting autoactivated GSK3 $\beta$," Journal of Neuroscience, vol. 30, no. 39, pp. 13138-13149, 2010.

[161] H. L. Melrose, J. C. Dächsel, B. Behrouz et al., "Impaired dopaminergic neurotransmission and microtubule-associated protein tau alterations in human LRRK2 transgenic mice," Neurobiology of Disease, vol. 40, no. 3, pp. 503-517, 2010.

[162] X. Lin, L. Parisiadou, X. L. Gu et al., "Leucine-rich repeat kinase 2 regulates the progression of neuropathology induced by Parkinson's-disease-related mutant $\alpha$-synuclein," Neuron, vol. 64, no. 6, pp. 807-827, 2009.

[163] T. Duka, V. Duka, J. N. Joyce, and A. Sidhu, " $\alpha$-synuclein contributes to GSK-3 $\beta$-catalyzed tau phosphorylation in Parkinson's disease models," Journal of the Federation of American Societies for Experimental Biology, vol. 23, no. 9, pp. 2820-2830, 2009.

[164] X. Zhu, A. Babar, S. L. Siedlak et al., "LRRK2 in Parkinson's disease and dementia with Lewy bodies," Molecular Neurodegeneration, vol. 1, no. 17, 2006.

[165] H. Qing, Y. Zhang, Y. Deng, E. G. McGeer, and P. L. McGeer, "Lrrk2 interaction with $\alpha$-synuclein in diffuse Lewy body disease," Biochemical and Biophysical Research Communications, vol. 390, no. 4, pp. 1229-1234, 2009.

[166] J. Simón-Sánchez, C. Schulte, J. M. Bras et al., "Genome-wide association study reveals genetic risk underlying Parkinson's disease," Nature Genetics, vol. 41, no. 12, pp. 1308-1312, 2009.

[167] W. Satake, Y. Nakabayashi, I. Mizuta et al., "Genome-wide association study identifies common variants at four loci as genetic risk factors for Parkinson's disease," Nature Genetics, vol. 41, no. 12, pp. 1303-1307, 2009.

[168] S. DiMauro and E. A. Schon, "Mitochondrial respiratorychain diseases," The New England Journal of Medicine, vol. 348, no. 26, pp. 2656-2668, 2003.

[169] C. Vives-Bauza and S. Przedborski, "Mitophagy: the latest problem for Parkinson's disease," Trends in Molecular Medicine, vol. 17, no. 3, pp. 158-165, 2011.

[170] N. Matsuda, S. Sato, K. Shiba et al., "PINK1 stabilized by mitochondrial depolarization recruits Parkin to damaged mitochondria and activates latent Parkin for mitophagy," Journal of Cell Biology, vol. 189, no. 2, pp. 211-221, 2010.

[171] D. P. Narendra, S. M. Jin, A. Tanaka et al., "PINK1 is selectively stabilized on impaired mitochondria to activate Parkin," Public Library of Science, Biology, vol. 8, no. 1, Article ID e1000298., 2010.

[172] J. Park, S. B. Lee, S. Lee et al., "Mitochondrial dysfunction in Drosophila PINK1 mutants is complemented by parkin," Nature, vol. 441, no. 7097, pp. 1157-1161, 2006.

[173] J. Y. Lee, Y. Nagano, J. P. Taylor, K. L. Lim, and T. P. Yao, "Disease-causing mutations in parkin impair mitochondrial ubiquitination, aggregation, and HDAC6-dependent mitophagy," Journal of Cell Biology, vol. 189, no. 4, pp. 671679, 2010.

[174] M. M. M. Wilhelmus, S. M. A. Van Der Pol, Q. Jansen et al., "Association of Parkinson disease-related protein PINK1 with Alzheimer disease and multiple sclerosis brain lesions," Free Radical Biology and Medicine, vol. 50, no. 3, pp. 469-476, 2011. 
[175] M. E. Witte, J. G. J. M. Bol, W. H. Gerritsen et al., "Parkinson's disease-associated parkin colocalizes with Alzheimer's disease and multiple sclerosis brain lesions," Neurobiology of Disease, vol. 36, no. 3, pp. 445-452, 2009.

[176] C. R. Scherzer, R. V. Jensen, S. R. Gullans, and M. B. Feany, "Gene expression changes presage neurodegeneration in a Drosophila model of Parkinson's disease," Human Molecular Genetics, vol. 12, no. 19, pp. 2457-2466, 2003.

[177] L. Lu, F. Neff, D. Alvarez-Fischer et al., "Gene expression profiling of Lewy body-bearing neurons in Parkinson's disease," Experimental Neurology, vol. 195, no. 1, pp. 27-39, 2005.

[178] M. Shi, W. M. Caudle, and J. Zhang, "Biomarker discovery in neurodegenerative diseases: a proteomic approach," Neurobiology of Disease, vol. 35, no. 2, pp. 157-164, 2009.

[179] J. Jin, G. E. Meredith, L. Chen et al., "Quantitative proteomic analysis of mitochondrial proteins: relevance to Lewy body formation and Parkinson's disease," Molecular Brain Research, vol. 134, no. 1, pp. 119-138, 2005.

[180] M. Basso, S. Giraudo, D. Corpillo, B. Bergamasco, L. Lopiano, and M. Fasano, "Proteome analysis of human substantia nigra in Parkinson's disease," Proteomics, vol. 4, no. 12, pp. 3943-3952, 2004.

[181] M. Shi, J. Jin, Y. Wang et al., "Mortalin: a protein associated with progression of Parkinson disease?" Journal of Neuropathology and Experimental Neurology, vol. 67, no. 2, pp. 117-124, 2008.

[182] J. B. Leverenz, I. Umar, Q. Wang et al., "Proteomic identification of novel proteins in cortical Lewy bodies," Brain Pathology, vol. 17, no. 2, pp. 139-145, 2007.

[183] F. Abdi, J. F. Quinn, J. Jankovic et al., "Detection of biomarkers with a multiplex quantitative proteomic platform in cerebrospinal fluid of patients with neurodegenerative disorders," Journal of Alzheimer's Disease, vol. 9, no. 3, pp. 293-348, 2006.

[184] K. Wada-Isoe, K. Michio, K. Imamura et al., "Serum proteomic profiling of dementia with Lewy bodies: diagnostic potential of SELDI-TOF MS analysis," Journal of Neural Transmission, vol. 114, no. 12, pp. 1579-1583, 2007.

[185] M. E. Bianchi, "DAMPs, PAMPs and alarmins: all we need to know about danger," Journal of Leukocyte Biology, vol. 81, no. 1, pp. $1-5,2007$. 


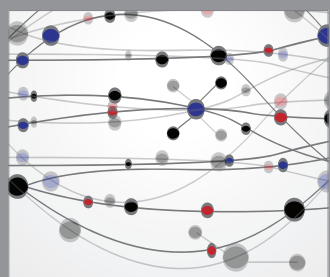

The Scientific World Journal
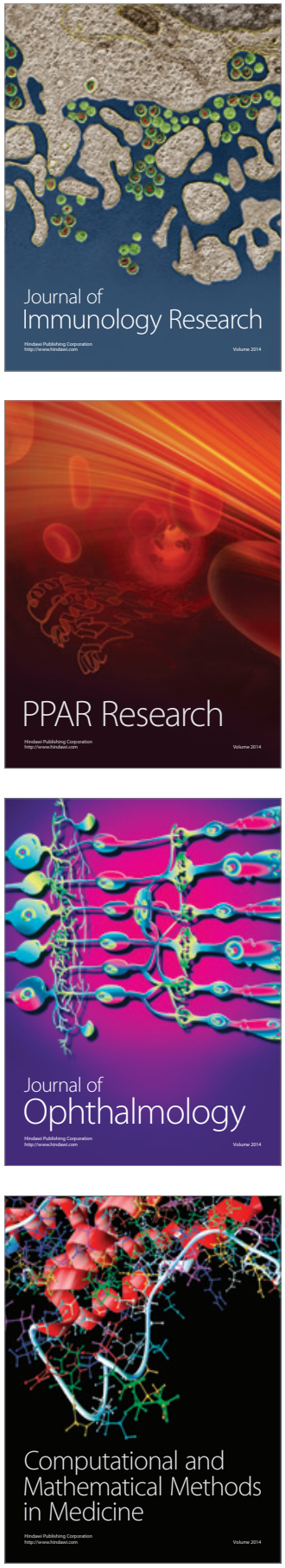

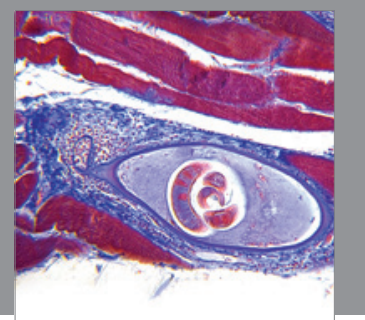

Gastroenterology

Research and Practice
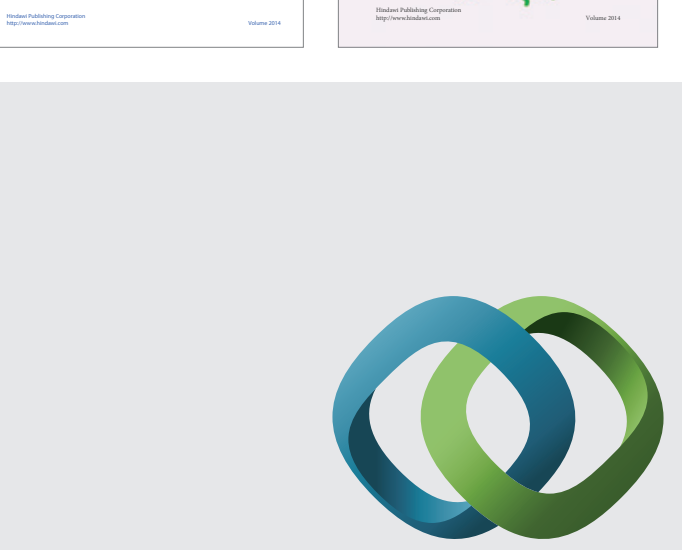

\section{Hindawi}

Submit your manuscripts at

http://www.hindawi.com
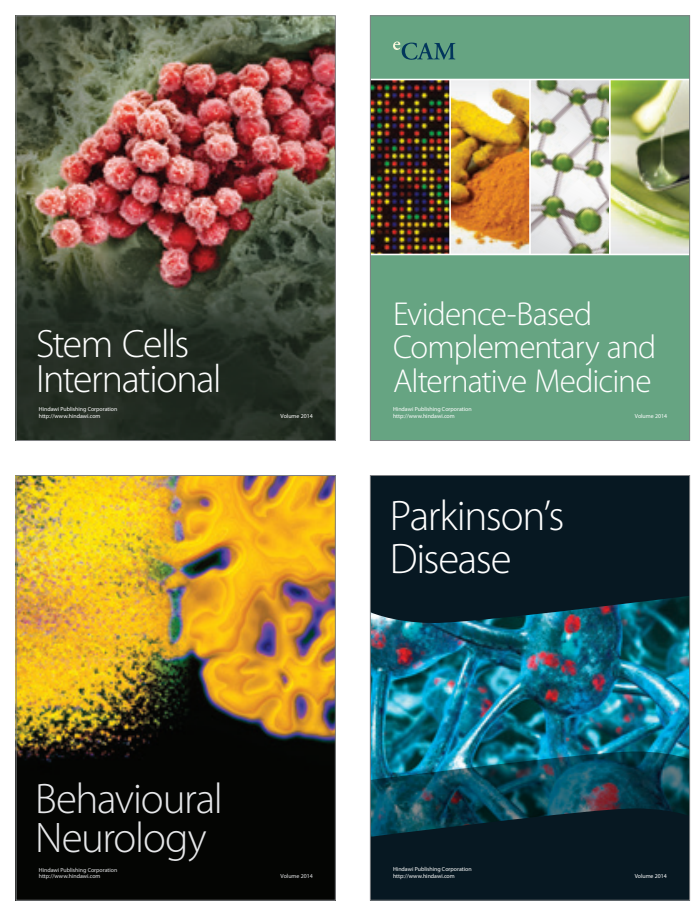

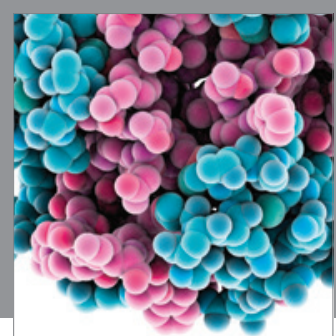

Journal of
Diabetes Research

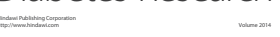

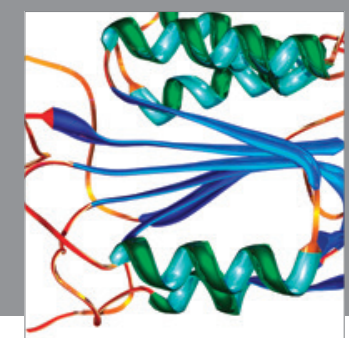

Disease Markers
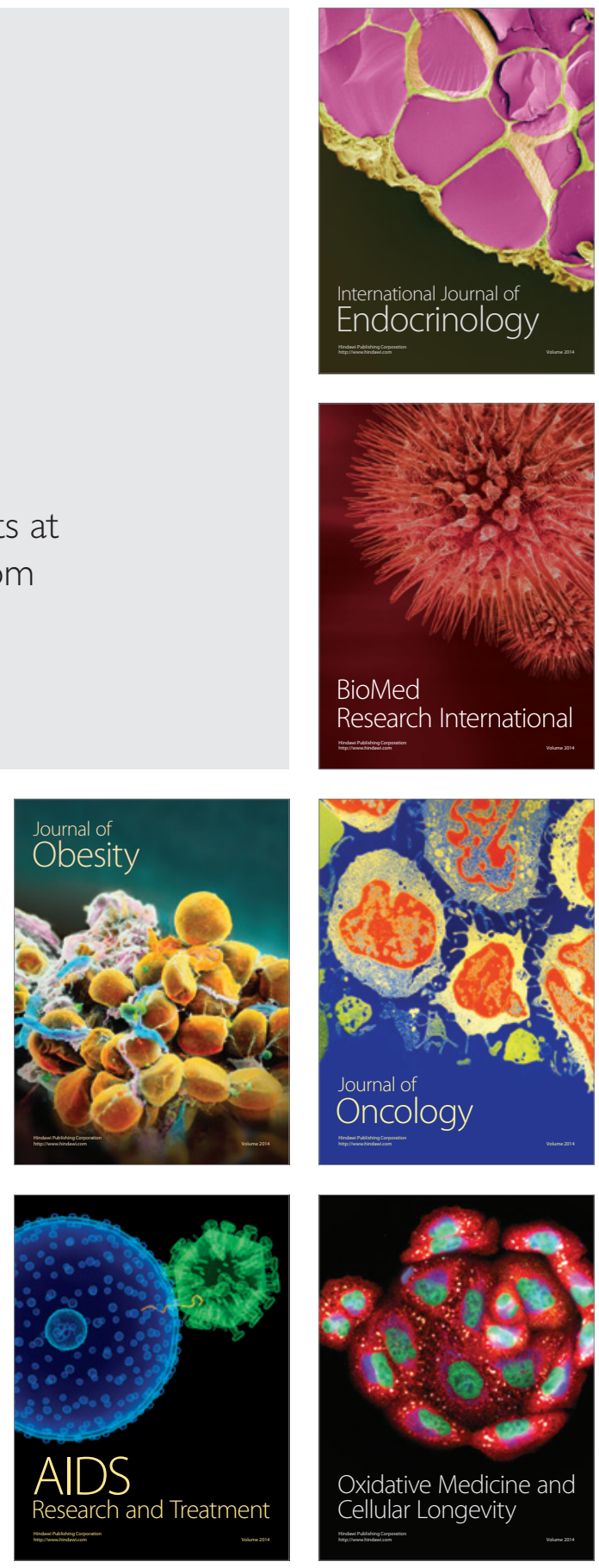